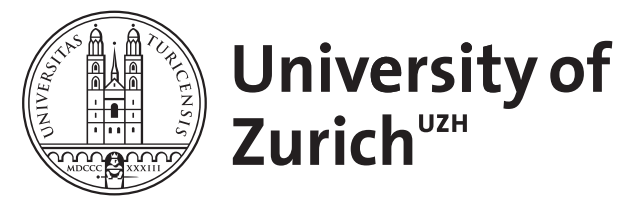

\title{
Coisotropic submanifolds and dual pairs
}

Cattaneo, Alberto S

\begin{abstract}
The Poisson sigma model is a widely studied two-dimensional topological field theory. This note shows that boundary conditions for the Poisson sigma model are related to coisotropic submanifolds (a result announced in [math.QA/0309180]) and that the corresponding reduced phase space is a (possibly singular) dual pair between the reduced spaces of the given two coisotropic submanifolds. In addition the generalization to a more general tensor field is considered and it is shown that the theory produces Lagrangian evolution relations if and only if the tensor field is Poisson.
\end{abstract}

DOI: https://doi.org/10.1007/s11005-013-0661-2

Posted at the Zurich Open Repository and Archive, University of Zurich ZORA URL: https://doi.org/10.5167/uzh-85296

Journal Article

Originally published at:

Cattaneo, Alberto S (2014). Coisotropic submanifolds and dual pairs. Letters in Mathematical Physics, 104(3):243-270.

DOI: https://doi.org/10.1007/s11005-013-0661-2 


\title{
Coisotropic Submanifolds and Dual Pairs
}

\author{
ALBERTO S. CATTANEO \\ Institut für Mathematik, Universität Zürich, Winterthurerstrasse 190, 8057 Zurich, \\ Switzerland. e-mail: alberto.cattaneo@math.uzh.ch
}

Received: 22 June 2013 / Revised: 11 October 2013 / Accepted: 12 October 2013

(C) Springer Science+Business Media Dordrecht 2013

\begin{abstract}
The Poisson sigma model is a widely studied two-dimensional topological field theory. This note shows that boundary conditions for the Poisson sigma model are related to coisotropic submanifolds (a result announced in [math.QA/0309180]) and that the corresponding reduced phase space is a (possibly singular) dual pair between the reduced spaces of the given two coisotropic submanifolds. In addition the generalization to a more general tensor field is considered and it is shown that the theory produces Lagrangian evolution relations if and only if the tensor field is Poisson.
\end{abstract}

Mathematics Subject Classification (1991). Primary 53D17; Secondary 81T45, 53D20, 58H05.

Keywords. coisotropic submanifolds, dual pairs, Poisson sigma model, Lagrangian field theories with boundary.

\section{Introduction}

Let $M$ be a finite-dimensional manifold and $I$ the unit interval $[0,1]$. We denote by $P M:=C^{1}(I, M)$ the space of differentiable paths on $M$ and by $T^{*} P M$ the space of bundle maps $T I \rightarrow T^{*} M$ with continuously differentiable base map and continuous fiber map. We consider $P M$ as a Banach manifold and $T^{*} P M$ as a Banach, weak symplectic manifold (a weak symplectic form is a closed 2-form that induces an injective map from the tangent to the cotangent bundle). The canonical symplectic form $\Omega$ is the differential of the canonical 1-form $\Theta$. If we denote an element of $T^{*} P M$ by $(X, \eta)$ where $X$ is the differentiable base map and the fiber map $\eta$ is regarded as a continuous section of $T^{*} I \otimes X^{*} T^{*} M$, we have

$$
\Theta(X, \eta)(\widehat{\xi})=\int_{I}\langle\eta, \xi\rangle,
$$

where $\widehat{\xi}$ is a tangent vector at $(X, \eta), \xi$ is its projection to $T_{X} P M=\Gamma\left(X^{*} T M\right)$, and $\langle$,$\rangle is the canonical pairing between the cotangent and the tangent bundles$ to $M$.

A.S. Cattaneo acknowledges partial support of SNF Grant No. 200020-131813/1. 
Let now $\pi$ be a section of $T M \otimes T M$. We denote by $\pi^{\sharp}$ the induced bundle map $T^{*} M \rightarrow T M$ satisfying

$$
\left\langle\pi^{\sharp}(x) \sigma, \tau\right\rangle=\pi(x)(\sigma, \tau), \quad \forall x \in M, \quad \forall \sigma, \tau \in T_{x}^{*} M .
$$

Given two submanifolds $C_{0}$ and $C_{1}$ of $M$, we denote by $\mathcal{C}_{\pi}\left(M ; C_{0}, C_{1}\right)$ the space of " $\pi$-compatible paths" from $C_{0}$ to $C_{1}$; i.e.,

$$
\mathcal{C}_{\pi}\left(M ; C_{0}, C_{1}\right):=\left\{(X, \eta) \in T^{*} P M: \mathrm{d} X+\pi^{\sharp}(X) \eta=0, X(0) \in C_{0}, \quad X(1) \in C_{1}\right\},
$$

where the differential $\mathrm{d} X$ of the base map is regarded as a section of $T^{*} I \otimes X^{*} T M$. By using the implicit function theorem, one can easily prove [6] that $\mathcal{C}_{\pi}(M ; M, M)$ is a Banach submanifold of $T^{*} P M$.

In general, for other submanifolds $C_{0}$ and $C_{1}, \mathcal{C}_{\pi}\left(M ; C_{0}, C_{1}\right)$ is not a Banach submanifold. ${ }^{1}$

Anyway, even if $\mathcal{C}_{\pi}\left(M ; C_{0}, C_{1}\right)$ is not a submanifold, we may define its Zarisky tangent space at each point. Namely, we first define the submanifold $T^{*} P M\left(C_{0}, C_{1}\right)$ of $T^{*} P M$ consisting of bundle maps whose base maps connect $C_{0}$ to $C_{1}$. Then the tangent space at $(X, \eta) \in \mathcal{C}_{\pi}\left(M ; C_{0}, C_{1}\right)$ is defined as the subspace of vectors in $T_{(X, \eta)} T^{*} P M\left(C_{0}, C_{1}\right)$ satisfying the linearized equation.

In general, we will call subvariety (of a smooth manifold) the common zero set of a family of smooth functions and, by abuse of notation, we will call tangent bundle the union of the Zarisky tangent spaces to a subvariety. Given a symplectic manifold $(\mathcal{M}, \omega)$ and a subvariety $\mathcal{C}$, we define, again by abuse of notation, the symplectic orthogonal bundle $T^{\perp} \mathcal{C}$ to $\mathcal{C}$ as the union $T_{x}^{\perp} \mathcal{C}, x \in \mathcal{C}$, with

$$
T_{x}^{\perp} \mathcal{C}:=\left\{v \in T_{x} \mathcal{M}: \omega_{x}(v, w)=0 \forall w \in T_{x} \mathcal{C}\right\} .
$$

The subvariety $\mathcal{C}$ is said to be coisotropic if $T^{\perp} \mathcal{C} \subset T C$.

\subsection{THE MAIN RESULTS}

In Section 2 we study the tangent bundle and the symplectic orthogonal bundle to $\mathcal{C}_{\pi}\left(M ; C_{0}, C_{1}\right)$. Relying on the results of Section 2, we prove in Section 3.1 (viz., Propositions 3.1 and 3.2) the following

THEOREM 1.1. $\mathcal{C}_{\pi}(M ; M, M)$ is coisotropic in $T^{*} P M$ iff $\pi$ is a Poisson bivector field.

Recall that a Poisson bivector field $\pi$ is a skew-symmetric 2-tensor field satisfying $[\pi, \pi]=0$, where $[$,$] is the Schouten-Nijenhuis bracket. A proof of the$

${ }^{1}$ The simplest example is when $\pi$ is identically zero. In this case $\mathcal{C}_{\pi}\left(M ; C_{0}, C_{1}\right)$ is a fibration over $B:=C_{0} \cap C_{1}$ with fiber at $x$ given by $\Omega^{1}\left(I, T_{x}^{*} M\right)$. If the basis $B$ is not a manifold, so neither is $\mathcal{C}_{\pi}\left(M ; C_{0}, C_{1}\right)$. 
if-part of the Theorem is contained in [6], but the result was already known [11,13] in the case when one considers loops instead of paths.

If $\mathcal{C}$ is a coisotropic submanifold of a weak symplectic Banach manifold, $T^{\perp} \mathcal{C}$ is an integrable distribution and the leaf space $\underline{\mathcal{C}}$, also called the reduced phase space, inherits a weak symplectic structure if it is a smooth manifold. The reduced phase space $\mathcal{C}_{\pi}(M ; M, M)$ has been shown in [6] to also have the structure of a topological groupoid; if it is smooth, it is a symplectic groupoid integrating the Poisson manifold $M$.

Now notice that not all possible boundary conditions allow solutions to the constraint equation. In other words, the maps

$$
p_{i}: \mathcal{C}_{\pi}\left(M ; C_{0}, C_{1}\right) \rightarrow C_{i}, \quad i=0,1,
$$

associating $X(i)$ to a solution $(X, \eta)$ may not be surjective. With this notation we may give the precise formulation, and a proof (see Section 3.2, in particular Propositions 3.5 and 3.6), of a result announced in [7] as Theorem 3.1 (implicitly assuming there that the $C_{i}$ s were chosen so that the $p_{i}$ s were surjective):

THEOREM 1.2. If $\pi$ is a Poisson bivector field, then $\mathcal{C}_{\pi}\left(M ; C_{0}, C_{1}\right)$ is coisotropic in $T^{*} P M$ iff $\operatorname{Im} p_{0}$ and $\operatorname{Im} p_{1}$ are coisotropic in $M$ relative to $C_{0}$ and $C_{1}$ respectively.

Recall that a submanifold $C$ of a finite-dimensional manifold $M$ endowed with a 2-tensor field $\pi$ is called coisotropic if $\pi^{\sharp}\left(N^{*} C\right) \subset T C$, where $N^{*} C$ denotes the conormal bundle to $C$ :

$$
N_{x}^{*} C:=\left\{\alpha \in T_{x}^{*} M:\langle\alpha, v\rangle=0 \forall v \in T_{x} C\right\}, \quad \forall x \in C .
$$

If $S$ is a subset of a submanifold $C$ of a Poisson manifold $M$, we say that $S$ is coisotropic in $M$ relative to $C$ if $\pi^{\sharp}\left(N_{x}^{*} C\right) \subset T_{x} C$ for all $x \in S$. We consider the empty set as a coisotropic submanifold of any symplectic or Poisson manifold.

Observe that having $\mathcal{C}_{\pi}\left(M ; C_{0}, C_{1}\right)$ coisotropic may not be enough to conclude that $\pi$ is Poisson as it is possible that the base paths contained in $\mathcal{C}_{\pi}\left(M ; C_{0}, C_{1}\right)$ do not explore the whole of $M .^{2}$

By definition we may also reformulate the if-part of Theorem 1.2 in the following (slightly weaker) form:

COROLLARY 1.3. If $\pi$ is Poisson and $C_{0}$ and $C_{1}$ are coisotropic, then $\mathcal{C}_{\pi}\left(M ; C_{0}, C_{1}\right)$ is coisotropic.

In this case, as recalled above, $T^{\perp} \mathcal{C}_{\pi}\left(M ; C_{0}, C_{1}\right)$ is an integrable distribution on $\mathcal{C}_{\pi}\left(M ; C_{0}, C_{1}\right)$, which we describe in details in Section 3.2.1.

${ }^{2}$ For example, assume that $\pi$ has a zero at a point $x$. Then $\mathcal{C}_{\pi}(M ;\{x\}, M)$ consists of pairs $(X, \eta)$ where $X$ is the constant path at $x$ and there are no conditions on $\eta$. It is then clear that $\mathcal{C}_{\pi}(M ;\{x\}, M)$ is coisotropic (actually Lagrangian) whatever the tensor $\pi$ is. 
In Section 4 we concentrate on the case when $C_{0}$ and $C_{1}$ are coisotropic submanifolds of a Poisson manifold $M$ and discuss how the reduction of $\mathcal{C}_{\pi}\left(M ; C_{0}, C_{1}\right)$ may be understood as a (singular) dual pair.

Finally in Section 5 we show that this reduction may also be recovered by Poisson reduction of the coisotropic submanifold of the symplectic groupoid of $M$ determined by the intersection of the preimages of $C_{0}$ and $C_{1}$ under the source and target maps, respectively.

Remark 1.4. One may consider the weaker condition that $\mathcal{C}_{\pi}\left(M ; C_{0}, C_{1}\right)$ be presymplectic (i.e., that the kernel of the restriction of the symplectic form is a subbundle of the tangent bundle of $\left.\mathcal{C}_{\pi}\left(M ; C_{0}, C_{1}\right)\right)$. As shown in [1], a sufficient condition for this to happen is that $C_{0}$ and $C_{1}$ are pre-Poisson submanifolds of $M$ (according to the definition in [8]). We will not elaborate on this in this paper.

\subsection{THE CASE OF THE CIRCLE}

In this paper we mainly work on the path space $P M$ as it is interesting to have boundary components (to be associated to the submanifolds $C_{0}$ and $C_{1}$ ). A very similar, actually a bit easier, story works in the case of the loop space $L M:=$ $C^{1}\left(S^{1}, M\right)$. In this case, we can analogously define the weak symplectic Banach manifold $T^{*} L M$ as the space of bundle maps $T S^{1} \rightarrow T^{*} M$ with continuously differentiable base map and continuous fiber map. The weak symplectic form $\Omega$ is again the differential of the canonical 1-form $\Theta$ :

$$
\Theta(X, \eta)(\widehat{\xi})=\int_{S^{1}}\langle\eta, \xi\rangle,
$$

where we use the same notations as above. To the tensor $\pi$ we now associate the Banach submanifold

$$
\mathcal{C}_{\pi}(M):=\left\{(X, \eta) \in T^{*} L M: \mathrm{d} X+\pi^{\sharp}(X) \eta=0\right\} .
$$

A very similar proof to Theorem 1.1, see Section 3.3, yields the following

THEOREM 1.5. $\mathcal{C}_{\pi}(M)$ is coisotropic in $T^{*} L M$ iff $\pi$ is a Poisson bivector field.

In this case, as recalled above, $T^{\perp} \mathcal{C}_{\pi}(M)$ is an integrable distribution on $\mathcal{C}_{\pi}(M)$, which we describe in details in Section 3.3.1.

\subsection{LAGRANGIAN FIELD THEORIES WITH BOUNDARY}

In [4] and [5] the general notion of Lagrangian field theories on manifolds with boundary is studied. The symplectic manifolds $T^{*} P M$ and $T^{*} L M$ described above arise as spaces of boundary fields of a two-dimensional Lagrangian field theory 
with $\mathcal{C}_{\pi}\left(M ; C_{0}, C_{1}\right)$ and $\mathcal{C}_{\pi}(M)$ as its "spaces of Cauchy data." The requirement of $\pi$ being Poisson turns out to be equivalent to the requirement that the theory is "good" in the sense that the the evolution relations determined by solutions to the Euler-Lagrange equations are (immersed) Lagrangian submanifolds. We discuss this in more details in Section 6.

\section{Tangent and Orthogonal Bundles}

By choosing a linear connection on $M$, which for simplicity we assume to be torsion free, we may identify the tangent bundle to $T^{*} M$ with the vector bundle $E:=T^{*} M \oplus T M \oplus T^{*} M$. Explicitly this is done as follows. First observe that both $T T^{*} M$ and $E$ can be regarded as vector bundles over $T^{*} M$ with fiber at point $(x, p)$ given by the vector space $T_{x} M \oplus T_{x}^{*} M$; the transition functions in the two vector bundles are however different. Choosing on $M$ local coordinates $\left\{x^{i}\right\}_{i=1, \ldots, m}, m=\operatorname{dim} M$, and the corresponding dual coordinates $\left\{p_{i}\right\}$ on $T_{x}^{*} M$, we consider the fiber isomorphism

$$
\begin{aligned}
\Phi_{(x, p)}: T_{(x, p)} T^{*} M=T_{x} M \oplus T_{x}^{*} M & \rightarrow E_{(x, p)}=T_{x} M \oplus T_{x}^{*} M \\
\left(\dot{x}^{i}, \dot{p}_{i}\right) & \mapsto\left(\dot{x}^{i}, \dot{p}_{i}-\Gamma_{s i}^{r}(x) p_{r} \dot{x}^{s}\right)
\end{aligned}
$$

where the $\Gamma$ s are the Christoffel symbols of the given connection, and we use Einstein's convention that a sum over upper and lower repeated indices is understood. Then $\Phi: T T^{*} M \rightarrow E$ is a vector bundle isomorphism.

By this we may also identify $T T^{*} P M$ with $T^{*} P M \oplus T P M \oplus T^{*} P M$, regarded as vector bundles over $T^{*} P M$. Recall that $T_{X} P M=\Gamma\left(X^{*} T M\right)$ and $T_{X}^{*} P M:=\Gamma\left(T^{*} I \otimes\right.$ $\left.X^{*} T^{*} M\right){ }^{3}$ To describe this isomorphism explicitly, we observe that, given a continuous path $X$, we may subdivide the interval $I$ into finitely many subintervals $I_{\alpha}$ such that $X\left(I_{\alpha}\right)$ is contained in a coordinate patch $\forall \alpha$. For a given $I_{\alpha}$, we denote by $X^{i}$ and $\eta_{i}, i=1, \ldots, m:=\operatorname{dim} M$, the components in local coordinates of the restrictions to $I_{\alpha}$ of $X$ and $\eta$. The restriction to $I_{\alpha}$ of a tangent vector $\widehat{\xi}$ can then be split correspondingly into its components $\xi^{i}$ in the $X^{i}$-direction and $\zeta_{i}$ in the $\eta_{i}$-direction. We then define

$$
e_{i}=\zeta_{i}-\Gamma_{s i}^{r}(X) \eta_{r} \xi^{s} .
$$

The map $\left(X^{i}, \eta_{i}, \xi^{i}, \zeta_{i}\right) \mapsto\left(X^{i}, \eta_{i}, \xi^{i}, e_{i}\right)$ is well-defined globally and yields the required vector bundle isomorphism $T T^{*} P M \rightarrow T^{*} P M \oplus T P M \oplus T^{*} P M$. If we now impose boundary conditions, by the same map we may finally identify the fiber at $(X, \eta) \in T^{*} P M\left(C_{0}, C_{1}\right)$ with

$$
\left\{\xi \oplus e \in \Gamma\left(X^{*} T M\right) \oplus \Gamma\left(T^{*} I \otimes X^{*} T^{*} M\right): \xi(0) \in T_{0}, \quad \xi(1) \in T_{1}\right\},
$$

\footnotetext{
${ }^{3}$ To avoid cumbersome notations, from now on we will avoid indicating which maps or forms are continuous or differentiable.
} 
with

$$
T_{0}:=T_{X(0)} C_{0} \quad \text { and } \quad T_{1}:=T_{X(1)} C_{1} .
$$

The linear connection on $M$ also induces a connection on the vector bundle $X^{*} T M$. We denote by $\partial: \Gamma\left(X^{*} T M\right) \rightarrow \Gamma\left(T^{*} I \otimes X^{*} T M\right)$ the corresponding covariant exterior derivative. In local coordinates, its action on a section $\sigma$ of $X^{*} T M$ is given by

$$
(\partial \sigma)^{i}=\mathrm{d} \sigma^{i}+\Gamma_{r s}^{i} \mathrm{~d} X^{r} \sigma^{s} .
$$

It is convenient to modify this connection by using

$$
A:=\nabla \pi^{\sharp}(X) \eta \in \Gamma\left(T^{*} I \otimes X^{*} \operatorname{End}(T M)\right),
$$

where $\nabla$ denotes the covariant derivative. In local coordinates we have

$$
(\nabla \pi)_{k}^{i j}=\partial_{k} \pi^{i j}+\Gamma_{k r}^{i} \pi^{r j}+\Gamma_{k r}^{j} \pi^{i r}
$$

and

$$
A_{k}^{i}=\eta_{j}(\nabla \pi)_{k}^{j i}(X)
$$

We will denote by $D$ the covariant exterior derivative $\partial+A$.

\subsection{THE TANGENT SPACES TO COMPATIBLE PATHS}

Let $(X, \eta)$ be a point in $\mathcal{C}_{\pi}\left(M ; C_{0}, C_{1}\right)$.

PROPOSITION 2.1. After choosing a connection on $M$ and using the above notations, we have

$$
\begin{aligned}
T_{(X, \eta)} \mathcal{C}_{\pi}\left(M ; C_{0}, C_{1}\right)= & \left\{\xi \in \Gamma\left(X^{*} T M\right), e \in \Gamma\left(T^{*} I \otimes X^{*} T^{*} M\right):\right. \\
& \left.(D \xi)+\pi^{\sharp}(X) e=0, \quad \xi(0) \in T_{0}, \quad \xi(1) \in T_{1}\right\} .
\end{aligned}
$$

Proof. Let us restrict our attention to a subinterval $I_{\alpha}$ such that $X\left(I_{\alpha}\right)$ is contained in a coordinate patch. The restriction to $I_{\alpha}$ of the equation satisfied by $X$ and $\eta$ then reads

$$
\mathrm{d} X^{i}+\eta_{j} \pi^{j i}(X)=0
$$

in local coordinates. Let $\xi^{i}$ and $\zeta_{i}$ denote the local-coordinate expression of a tangent vector. They then satisfy the equation

$$
\mathrm{d} \xi^{i}+\eta_{j} \partial_{l} \pi^{j i}(X) \xi^{l}+\zeta_{j} \pi^{j i}(X)=0,
$$


or equivalently by (2.4)

$$
\left(\mathrm{d} \xi^{i}-\xi^{l} \Gamma_{l r}^{i}(X) \eta_{j} \pi^{j r}\right)+\eta_{j}(\nabla \pi)_{l}^{j i}(X) \xi^{l}+\left(\zeta_{j}-\Gamma_{l j}^{s}(X) \eta_{s} \xi^{l}\right) \pi^{j i}(X)=0 .
$$

Since the connection is torsion free, by (2.6), (2.1), (2.2), (2.3) and (2.5) we conclude the proof.

We then consider the parallel transport $U \in \Gamma\left(\operatorname{Iso}\left(X^{*} T M, T_{X(0)} M\right)\right)$ of the connection $D$, viz., the solution to the Cauchy problem

$$
\left\{\begin{array}{l}
\mathrm{d} \circ U=U \circ D \\
U(0)=I d
\end{array}\right.
$$

In local coordinates, we may also write

$$
\left\{\begin{aligned}
\mathrm{d} U_{j}^{i} & =U_{l}^{i}\left(A_{j}^{l}+\Gamma_{s j}^{l} \mathrm{~d} X^{s}\right), \\
U(0)_{j}^{i} & =\delta_{j}^{i} .
\end{aligned}\right.
$$

We may then simplify the equation satisfied by the tangent vector $(\xi, e)$ into

$$
\mathrm{d} \lambda+P^{\sharp} \phi=0,
$$

with

$$
\begin{aligned}
\lambda & :=U \xi \in \Omega^{0}\left(I, T_{X(0)} M\right), \\
\phi & :=\left(U^{t}\right)^{-1} e \in \Omega^{1}\left(I, T_{X(0)}^{*} M\right), \\
P^{\sharp} & :=U \pi^{\sharp} U^{t} \in \Omega^{0}\left(I, \operatorname{Hom}\left(T_{X(0)}^{*} M, T_{X(0)} M\right)\right) .
\end{aligned}
$$

So we get

$$
\begin{aligned}
& T_{(X, \eta)} \mathcal{C}_{\pi}\left(M ; C_{0}, C_{1}\right) \cong T_{(X, \eta)} \mathcal{C}_{\pi}\left(M ; C_{0}, C_{1}\right)^{\mathrm{twisted}} \\
& \quad:=\left\{\lambda \in \Omega^{0}\left(I, T_{X(0)} M\right), \phi \in \Omega^{1}\left(I, T_{X(0)}^{*} M\right): \mathrm{d} \lambda+P^{\sharp} \phi=0,\right. \\
& \left.\quad \lambda(0) \in T_{0}, \quad U(1)^{-1} \lambda(1) \in T_{1}\right\} .
\end{aligned}
$$

Equation (2.9) may be easily solved for any $\phi$ just assigning the initial condition $\lambda(0)=\lambda_{0}$ :

$$
\lambda(u)=\lambda_{0}-\int_{0}^{u} P^{\sharp} \phi .
$$

Se we get the alternative description

$$
\begin{aligned}
T_{(X, \eta)} \mathcal{C}_{\pi}\left(M ; C_{0}, C_{1}\right) \cong T_{(X, \eta)} \mathcal{C}_{\pi}\left(M ; C_{0}, C_{1}\right)_{0} \\
\quad:=\left\{\lambda_{0} \in T_{0}, \phi \in \Omega^{1}\left(I, T_{X(0)}^{*} M\right): U(1)^{-1}\left(\lambda_{0}-\int_{I} P^{\sharp} \phi\right) \in T_{1}\right\} .
\end{aligned}
$$




\subsubsection{Properties of $P$}

The tensor $\pi$ has been replaced by $P$ in (2.10c). Just by differentiating, it is not difficult to see that $P^{\sharp}$ is the solution to the Cauchy problem

$$
\left\{\begin{aligned}
\mathrm{d} P^{\sharp} & =U T^{\sharp} U^{t}, \\
P^{\sharp}(0) & =\pi_{0}^{\sharp},
\end{aligned}\right.
$$

with $\pi_{0}:=\pi(X(0))$ and $T^{\sharp}:=D \pi^{\sharp}$. Using (2.6), (2.5) and (2.8), we obtain in local coordinates

$$
T^{l s}=\eta_{k}\left(\pi^{r s}(X)(\nabla \pi)_{r}^{k l}(X)-\pi^{k r}(X)(\nabla \pi)_{r}^{l s}(X)+\pi^{l r}(X)(\nabla \pi)_{r}^{k s}(X)\right) .
$$

Recall that, in local coordinates, the vanishing of the Schouten-Nijenhuis bracket of a bivector field $\pi$ may also be written, by using any connection, as

$$
\pi^{s r}(\nabla \pi)_{r}^{l k}+\pi^{k r}(\nabla \pi)_{r}^{s l}+\pi^{l r}(\nabla \pi)_{r}^{k s}=0 .
$$

This immediately implies the following

LEMMA 2.2. If $\pi$ is a Poisson bivector field, then $P=\pi_{0}$.

Observe that $P$ depends on the chosen $(X, \eta)$. We also have the following

LEMMA 2.3. If $P$ is skew-symmetric and constant for all $(X, \eta) \in \mathcal{C}_{\pi}(M ; M, M)$, then $\pi$ is a Poisson bivector field.

Proof. If $P$ is skew symmetric, then so is $\pi_{0}$, that is, $\pi$ at any possible starting point of a path $X$. Thus, $\pi$ is a bivector field. Moreover, for any $x \in M$, we can choose a solution to (2.6) with $X$ passing through $x$ for some $u_{0} \in I$ and, in a neighborhood of $u_{0}, \eta_{j}=\mathbf{e}_{j} \mathrm{~d} u$, with $\mathbf{e}_{j}$ a basis element of $\left(\mathbb{R}^{m}\right)^{*}$. This implies that $T$ vanishes at $u_{0}$ and hence that

$$
\pi^{r s}(x)(\nabla \pi)_{r}^{k l}(x)-\pi^{k r}(x)(\nabla \pi)_{r}^{l s}(x)+\pi^{l r}(x)(\nabla \pi)_{r}^{k s}(x)=0 .
$$

Since this holds for all $x \in M$, it follows that $\pi$ is Poisson.

\subsection{THE SYMPLECTIC ORTHOGONAL SPACES TO COMPATIBLE PATHS}

Assuming that the chosen connection is torsion-free, the symplectic form $\Omega$ evaluated at tangent vectors $(\xi, \zeta)$ and $(\widetilde{\xi}, \widetilde{\zeta})$ to $T^{*} P M$ at a point $(X, \eta)$ reads

$$
\Omega_{(X, \eta)}((\xi, \zeta),(\widetilde{\xi}, \widetilde{\zeta}))=\int_{I}\langle e, \widetilde{\xi}\rangle-\langle\widetilde{e}, \xi\rangle,
$$


where $(\xi \oplus e)$ and $(\widetilde{\xi} \oplus \widetilde{e})$ are the corresponding elements of $T_{X} P M \oplus T_{X}^{*} P M$. By the transformation (2.10a) and (2.10b), and the analogous ones $\widetilde{\lambda}=U \widetilde{\xi}, \widetilde{\phi}=$ $\left(U^{t}\right)^{-1} \widetilde{e}$, we get

$$
\Omega_{(X, \eta)}((\xi, \zeta),(\widetilde{\xi}, \widetilde{\zeta}))=\int_{I}\langle\phi, \tilde{\lambda}\rangle-\langle\widetilde{\phi}, \lambda\rangle
$$

Assume now that $(\xi, e)$ is tangent to $\mathcal{C}_{\pi}\left(M ; C_{0}, C_{1}\right)$. Then, by (2.12),

$$
\begin{aligned}
\int_{I}\langle\widetilde{\phi}, \lambda\rangle & =\left\langle\int_{I} \widetilde{\phi}, \lambda_{0}\right\rangle-\int_{I}\left\langle\widetilde{\phi}, \int_{0} P^{\sharp} \phi\right\rangle \\
& =\left\langle\int_{I} \tilde{\phi}, \lambda_{0}\right\rangle-\int_{I}\left\langle\phi,\left(P^{\sharp}\right)^{t} \int_{\bullet}^{1} \tilde{\phi}\right\rangle .
\end{aligned}
$$

We thus obtain

$$
\begin{aligned}
& T_{(X, \eta)}^{\perp} \mathcal{C}_{\pi}\left(M ; C_{0}, C_{1}\right) \cong T_{(X, \eta)}^{\perp} \mathcal{C}_{\pi}\left(M ; C_{0}, C_{1}\right)^{\text {implicit }} \\
& :=\left\{\tilde{\lambda} \in \Omega^{0}\left(I, T_{X(0)} M\right), \widetilde{\phi} \in \Omega^{1}\left(I, T_{X(0)}^{*} M\right):\right. \\
& \left.\int_{I}\left\langle\phi, \widetilde{\lambda}+\left(P^{\sharp}\right)^{t} \int_{0}^{1} \widetilde{\phi}\right\rangle-\left\langle\int_{I} \widetilde{\phi}, \lambda_{0}\right\rangle=0, \quad \forall\left(\lambda_{0}, \phi\right) \in T_{(X, \eta)} \mathcal{C}_{\pi}\left(M ; C_{0}, C_{1}\right)_{0}\right\} .
\end{aligned}
$$

\section{Proofs to the Main Theorems}

Using the results and notations of Section 2, we are now going to prove the main Theorems 1.1 and 1.2 and to draw further consequences.

\subsection{PROOF OF THEOREM 1.1}

In the case $C_{0}=C_{1}=M$, we have

$$
T_{(X, \eta)} \mathcal{C}_{\pi}(M ; M, M)_{0}:=\left\{\lambda_{0} \in T_{X(0)} M, \phi \in \Omega^{1}\left(I, T_{X(0)}^{*} M\right)\right\} .
$$

Thus, $(\widetilde{\lambda}, \widetilde{\phi})$ belongs to $T_{(X, \eta)}^{\perp} \mathcal{C}_{\pi}(M ; M, M)^{\text {implicit }}$ iff

$$
\int_{I}\left\langle\phi, \widetilde{\lambda}+\left(P^{\sharp}\right)^{t} \int_{\cdot}^{1} \widetilde{\phi}\right\rangle-\left\langle\int_{I} \widetilde{\phi}, \lambda_{0}\right\rangle=0
$$

for all $\lambda_{0} \in T_{X(0)} M$ and $\phi \in \Omega^{1}\left(I, T_{X(0)}^{*} M\right)$. This implies that $(\widetilde{\lambda}, \widetilde{\phi})$ belongs to $T_{(X, \eta)}^{\perp} \mathcal{C}_{\pi}(M ; M, M)^{\text {implicit }}$ iff 


$$
\widetilde{\lambda}(u)+\left(P^{\sharp}\right)^{t} \int_{u}^{1} \widetilde{\phi}=0
$$

and

$$
\int_{I} \widetilde{\phi}=0 .
$$

Now we have

PROPOSITION 3.1. If $\pi$ is a Poisson bivector field, then $\mathcal{C}_{\pi}(M ; M, M)$ is coisotropic.

Proof. By Lemma 2.2 we have $P=\pi_{0}$. So (3.1) implies that $(\widetilde{\lambda}, \widetilde{\phi})$ belongs to $T_{(X, \eta)} \mathcal{C}_{\pi}(M ; M, M)^{\text {twisted }}$. Thus, $\mathcal{C}_{\pi}(M ; M, M)$ is coisotropic.

PROPOSITION 3.2. If $\mathcal{C}_{\pi}(M ; M, M)$ is coisotropic, then $\pi$ is a Poisson bivector field.

Proof. Since $T_{(X, \eta)}^{\perp} \mathcal{C}_{\pi}(M ; M, M) \subset T_{(X, \eta)} \mathcal{C}_{\pi}(M ; M, M)$, any pair $(\tilde{\lambda}, \widetilde{\phi})$ satisfying (3.1) and (3.2) also belongs to $T_{(X, \eta)} \mathcal{C}_{\pi}(M ; M, M)^{\text {twisted; i.e., it satisfies }}$

$$
\mathrm{d} \widetilde{\lambda}+P^{\sharp} \widetilde{\phi}=0 .
$$

On the other hand, differentiating (3.1) yields

$$
\mathrm{d} \widetilde{\lambda}+\mathrm{d}\left(P^{\sharp}\right)^{t} \int_{\cdot}^{1} \widetilde{\phi}-\left(P^{\sharp}\right)^{t} \widetilde{\phi}=0 .
$$

So we get,

$$
\mathrm{d}\left(P^{\sharp}\right)^{t} \int_{\bullet}^{1} \widetilde{\phi}_{j}-\left(\left(P^{\sharp}\right)^{t}+P^{\sharp}\right) \widetilde{\phi}=0
$$

for any $\widetilde{\phi}$ satisfying (3.2). Now let $u_{0}<u_{1}$ be points on $I$. Let $U_{0}$ and $U_{1}$ be disjoint neighborhoods of $u_{0}$ and $u_{1}$ with $U_{0}<U_{1}$. We then choose $\widetilde{\phi}$ to vanish outside $U_{0} \cup U_{1}$. For $U_{0}<u<U_{1}$, (3.3) yields

$$
\mathrm{d}\left(P^{\sharp}\right)^{t}(u) \sigma=0
$$

with $\sigma:=\int_{u}^{1} \widetilde{\phi}$. Since this holds for all $\sigma \in T_{X(0)}^{*} M$, we see that $P$ must be constant. So now (3.3) reads

$$
\left(\left(P^{\sharp}\right)^{t}+P^{\sharp}\right) \widetilde{\phi}=0 \text {. }
$$


Again this must hold for all $\widetilde{\phi}$ satisfying (3.2). From this we conclude that $P$ must be skew-symmetric. Since these conclusions must hold for any solution $(X, \eta)$, Lemma 2.3 completes the proof.

This concludes the proof of Theorem 1.1.

\subsection{PROOF OF THEOREM 1.2}

Assuming that $\pi$ is a Poisson bivector field, we know by Lemma 2.2 that $P$ is constant and equal to $\pi_{0}=\pi(X(0))$. So $(\widetilde{\lambda}, \widetilde{\phi})$ belongs to $T_{(X, \eta)}^{\perp} \mathcal{C}_{\pi}\left(M ; C_{0}, C_{1}\right)^{\text {implicit }}$ iff

$$
\int_{I}\left\langle\phi, \tilde{\lambda}-\pi_{0}^{\sharp} \int^{1} \tilde{\phi}\right\rangle-\left\langle\int_{I} \tilde{\phi}, \lambda_{0}\right\rangle=0
$$

for all $\lambda_{0} \in T_{0}$ and $\phi \in \Omega^{1}\left(I, T_{X(0)}^{*} M\right)$ such that

$$
U(1)^{-1}\left(\lambda_{0}-\pi_{0}^{\#} \int_{I} \phi\right) \in T_{1} .
$$

PROPOSITION 3.3. Let $N_{i}^{*}=N_{X(i)}^{*} C_{i}, i=1$, 2. If $\pi$ is Poisson, then

$$
\begin{aligned}
& T_{(X, \eta)}^{\perp} \mathcal{C}_{\pi}\left(M ; C_{0}, C_{1}\right)^{\text {implicit }}=T_{(X, \eta)}^{\perp} \mathcal{C}_{\pi}\left(M ; C_{0}, C_{1}\right)^{\text {explicit }} \\
& :=\left\{\widetilde{\lambda} \in \Omega^{0}\left(I, T_{X(0)} M\right), \widetilde{\phi} \in \Omega^{1}\left(I, T_{X(0)}^{*} M\right): \mathrm{d} \widetilde{\lambda}+\pi_{0}^{\sharp} \widetilde{\phi}=0,\right. \\
& \left.\quad \widetilde{\lambda}(0) \in \pi_{0}^{\sharp}\left(N_{0}^{*}\right), U(1)^{-1} \widetilde{\lambda}(1) \in \pi_{1}^{\sharp}\left(N_{1}^{*}\right)\right\} .
\end{aligned}
$$

Proof. We may first consider $\lambda_{0}=0$ and $\phi$ such that $\int_{I} \phi=0$. Since (3.4) must hold in particular for all $\left(\lambda_{0}, \phi\right)$ of this kind, we obtain that there must be a constant $\tilde{\lambda}_{1} \in T_{X(0)} M$ such that

$$
\tilde{\lambda}(u)-\pi_{0}^{\sharp} \int_{u}^{1} \widetilde{\phi}=\tilde{\lambda}_{1}, \quad \forall u \in I .
$$

So (3.4) simplifies to

$$
\left\langle\int_{I} \phi, \widetilde{\lambda}_{1}\right\rangle-\left\langle\int_{I} \widetilde{\phi}, \lambda_{0}\right\rangle=0 .
$$

Observe now that $\left\langle\int_{I} \phi, \widetilde{\lambda}_{1}\right\rangle=\left\langle U(1)^{t} \int_{I} \phi, U(1)^{-1} \widetilde{\lambda}_{1}\right\rangle$. Set $\pi_{1}=\pi(X(1))$. Since

$$
U(1) \pi_{1}^{\sharp} U(1)^{t}=P(1)^{\sharp}=\pi_{0}^{\sharp},
$$


we have that $U(1)^{-1} \pi_{0}^{\sharp} \int_{I} \phi=\pi_{1}^{\sharp} U(1)^{t} \int_{I} \phi$. By choosing again $\lambda_{0}=0$, we get the condition

$$
\begin{aligned}
& \left\langle U(1)^{t} \int_{I} \phi, U(1)^{-1} \widetilde{\lambda}_{1}\right\rangle=0, \\
& \forall \phi \in \Omega^{1}\left(I, T_{X(0)}^{*} M\right) \text { such that } \pi_{1}^{\sharp} U(1)^{t} \int_{I} \phi \in T_{1} .
\end{aligned}
$$

Thus,

$$
\left\langle\alpha, U(1)^{-1} \widetilde{\lambda}_{1}\right\rangle=0, \quad \forall \alpha \in T_{X(1)}^{*} M \text { such that } \pi_{1}^{\sharp} \alpha \in T_{1} .
$$

We use now the following simple fact from linear algebra:

LEMMA 3.4. Let $V$ and $W$ be vector spaces. Let $F$ be a linear map $V \rightarrow W$ and $T$ a linear subspace of $W$. Then

$$
\operatorname{Ann}\left(F^{-1}(T)\right)=F^{t}(\operatorname{Ann}(T)),
$$

where Ann denotes the annihilator of a subspace (e.g., $\operatorname{Ann}(T)=\left\{\tau \in W^{*}: \tau(t)=\right.$ $0 \forall t \in T\}$ ).

Proof. It is obvious that $F^{t}(\operatorname{Ann}(T)) \subset \operatorname{Ann}\left(F^{-1}(T)\right)$. We now prove the other inclusion. Let $V^{\prime}$ be a complement of $F^{-1}(T)$ in $V$ and $W^{\prime}$ a complement of $T \oplus F\left(V^{\prime}\right)$ in $W$. Since the restriction $\left.F\right|_{V^{\prime}}$ of $F$ to $V^{\prime}$ establishes an isomorphism between $V^{\prime}$ and $F\left(V^{\prime}\right)$, for any $\psi \in \operatorname{Ann}\left(F^{-1}(T)\right) \subset V^{*}$ there is a unique $\phi \in F\left(V^{\prime}\right)^{*}$ with $\psi=\left.F\right|_{V^{\prime}} ^{t}(\phi)$. Now let $\varphi \in W^{*}$ be equal to $\phi$ when evaluated on elements of $F\left(V^{\prime}\right)$ and zero when evaluated on elements of $T$ or $W^{\prime}$. So $\varphi \in \operatorname{Ann}(T)$. Since $\psi=F^{t}(\varphi)$, this concludes the proof.

We apply the Lemma to (3.9) with $V=T_{X(1)}^{*} M, W=T_{X(1)} M, T=T_{1}$ and $F=\pi_{1}^{\sharp}$. Since $\pi_{1}$ is skew-symmetric, $F^{t}=-\pi_{1}^{\sharp}$. So we get that necessarily

$$
U(1)^{-1} \widetilde{\lambda}_{1} \in \pi_{1}^{\sharp} N_{1}^{*},
$$

where $N_{1}^{*}=N_{X(1)}^{*} C$ is the annihilator of $T_{1}$. So there exists $\theta \in N_{1}^{*}$ such that $\tilde{\lambda}_{1}=$ $U(1) \pi_{1}^{\sharp} \theta$, and we may rewrite (3.7) as

$$
\left\langle\int_{I} \phi, U(1) \pi_{1}^{\sharp} \theta\right\rangle-\left\langle\int_{I} \tilde{\phi}, \lambda_{0}\right\rangle=0,
$$

or equivalently, using again (3.8) and the skew-symmetry of $\pi$,

$$
\left\langle\theta, U(1)^{-1} \pi_{0}^{\sharp} \int_{I} \phi\right\rangle+\left\langle\int_{I} \widetilde{\phi}, \lambda_{0}\right\rangle=0 .
$$


This equation has to be satisfied for all $\lambda_{0} \in T_{0}$ and $\phi \in \Omega^{1}\left(I, T_{X(0)}^{*} M\right)$ satisfying (3.5). This is equivalent to imposing

$$
\left\langle\left(U(1)^{t}\right)^{-1} \theta+\int_{I} \widetilde{\phi}, \lambda_{0}\right\rangle=0
$$

for all $\lambda_{0} \in T_{0}$. That is,

$$
\left(U(1)^{t}\right)^{-1} \theta+\int_{I} \widetilde{\phi} \in N_{0}^{*},
$$

where $N_{0}^{*}=N_{X(0)}^{*} C_{0}$ is the annihilator of $T_{0}$. Recalling (3.6),

$$
\widetilde{\lambda}(u)-\pi_{0}^{\sharp} \int_{u}^{1} \widetilde{\phi}=\widetilde{\lambda}_{1}=U(1) \pi_{1}^{\sharp} \theta=\pi_{0}^{\sharp}\left(U^{t}\right)^{-1} \theta, \quad \forall u \in I,
$$

we see that a pair $(\widetilde{\lambda}, \widetilde{\phi})$ belongs to $T_{(X, \eta)}^{\perp} \mathcal{C}_{\pi}\left(M ; C_{0}, C_{1}\right)^{\text {implicit }}$ iff there exists $\theta \in N_{1}^{*}$ such that (3.11) and

$$
\tilde{\lambda}(u)=\pi_{0}^{\sharp}\left(\left(U^{t}\right)^{-1} \theta+\int_{u}^{1} \widetilde{\phi}\right), \quad \forall u \in I,
$$

are satisfied. By differentiating, in order to get rid of $\theta$, we finally obtain

$$
T_{(X, \eta)}^{\perp} \mathcal{C}_{\pi}\left(M ; C_{0}, C_{1}\right)^{\text {implicit }} \subset T_{(X, \eta)}^{\perp} \mathcal{C}_{\pi}\left(M ; C_{0}, C_{1}\right)^{\text {explicit }} .
$$

To prove the other inclusion, consider a pair $(\widetilde{\lambda}, \widetilde{\phi}) \in T_{(X, \eta)}^{\perp} \mathcal{C}_{\pi}\left(M ; C_{0}, C_{1}\right)^{\text {explicit }}$. Since $U(1)^{-1} \widetilde{\lambda}(1) \in \pi_{1}^{\sharp}\left(N_{1}^{*}\right)$, there exists $\theta \in N_{1}^{*}$ such that $\widetilde{\lambda}_{1}=U(1) \pi_{1}^{\sharp} \theta$. Then the solution to the equation has the form in (3.13) and satisfies (3.11).

PROPOSITION 3.5. Assume $\pi$ to be Poisson. If $C_{0}$ and $C_{1}$ are coisotropic, then so is $\mathcal{C}_{\pi}\left(M ; C_{0}, C_{1}\right)$.

Proof. In this case, by (2.11), we immediately have

$$
\begin{aligned}
& T_{(X, \eta)}^{\perp} \mathcal{C}_{\pi}\left(M ; C_{0}, C_{1}\right)^{\text {explicit }} \subset T_{(X, \eta)} \mathcal{C}_{\pi}\left(M ; C_{0}, C_{1}\right)^{\text {twisted }} \\
& =\left\{\lambda \in \Omega^{0}\left(I, T_{X(0)} M\right), \phi \in \Omega^{1}\left(I, T_{X(0)}^{*} M\right): \mathrm{d} \lambda+\pi_{0}^{\sharp} \phi=0,\right. \\
& \left.\quad \lambda(0) \in T_{0}, \quad U(1)^{-1} \lambda(1) \in T_{1}\right\},
\end{aligned}
$$

for all $(X, \eta) \in \mathcal{C}_{\pi}\left(M ; C_{0}, C_{1}\right)$. So $\mathcal{C}_{\pi}\left(M ; C_{0}, C_{1}\right)$ is coisotropic.

PROPOSITION 3.6. Assume $\pi$ to be Poisson. If $\mathcal{C}_{\pi}\left(M ; C_{0}, C_{1}\right)$ is coisotropic, the so are $\operatorname{Im} p_{0}$ and $\operatorname{Im} p_{1}$. 
Proof. If $\mathcal{C}_{\pi}\left(M ; C_{0}, C_{1}\right)$ is coisotropic, then any pair $(\widetilde{\lambda}, \widetilde{\phi})$ that belongs to $T_{(X, \eta)}^{\perp}$ $\mathcal{C}_{\pi}\left(M ; C_{0}, C_{1}\right)^{\text {explicit }}$ must also belong to $T_{(X, \eta)} \mathcal{C}_{\pi}\left(M ; C_{0}, C_{1}\right)^{\text {twisted }} \forall(X, \eta) \in \mathcal{C}_{\pi}$ $\left(M ; C_{0}, C_{1}\right)$. Thus, in particular, we must have $\widetilde{\lambda}(0) \in T_{0}$ and $U(1)^{-1} \widetilde{\lambda}(1) \in T_{1}$.

We may arbitrarily choose the end condition $\widetilde{\lambda}(1)$ such that $U(1)^{-1} \widetilde{\lambda}(1) \in \pi_{1}^{\sharp}\left(N_{1}^{*}\right)$ since condition (3.11) will always be satisfied by an appropriate choice of $\widetilde{\phi}$ (e.g., such that $\left.\int_{I} \widetilde{\phi}=-\left(U(1)^{t}\right)^{-1} \theta\right)$. So we see that $\pi_{1}^{\sharp}\left(N_{1}^{*}\right) \subset T_{1}$. Since this must hold for all $(X, \eta) \in \mathcal{C}_{\pi}\left(M ; C_{0}, C_{1}\right)$, we obtain that $N_{x}^{*} C_{1} \subset T_{x} C_{1} \forall x \in \operatorname{Im} p_{1}$.

Similarly, we may arbitrarily choose $\widetilde{\lambda}(0) \in \pi_{0}^{\sharp}\left(N_{0}^{*}\right)$ since the condition $U(1)^{-1} \widetilde{\lambda}(1)$ $\in \pi_{1}^{\sharp}\left(N_{1}^{*}\right)$ will always be satisfied by an appropriate choice of $\widetilde{\phi}$ (e.g., such that $\int_{I} \widetilde{\phi}=\tau$ if $\left.\tilde{\lambda}(0)=\pi_{0}^{\sharp} \tau\right)$. Then we see that $\pi_{0}^{\sharp}\left(N_{0}^{*}\right) \subset T_{0}$ and, since this must hold for all $(X, \eta) \in \mathcal{C}_{\pi}\left(M ; C_{0}, C_{1}\right)$, we obtain that $N_{x}^{*} C_{0} \subset T_{x} C_{0} \forall x \in \operatorname{Im} p_{0}$.

This concludes the proof of Theorem 1.2.

\subsubsection{Symmetries}

From now on we assume that $\pi$ is Poisson and that $C_{0}$ and $C_{1}$ are coisotropic. By defining

$$
b:=\left(U(1)^{t}\right)^{-1} \theta+\int_{\bullet}^{1} \widetilde{\phi} \in \Omega^{0}\left(I, T_{X(0)}^{*} M\right),
$$

we finally obtain

$$
\begin{aligned}
& T_{(X, \eta)}^{\perp} \mathcal{C}_{\pi}\left(M ; C_{0}, C_{1}\right)=T_{(X, \eta)}^{\perp} \mathcal{C}_{\pi}\left(M ; C_{0}, C_{1}\right)^{\text {explicit }} \\
& \quad:=\left\{\widetilde{\lambda} \in \Omega^{0}\left(I, T_{X(0)} M\right), \widetilde{\phi} \in \Omega^{1}\left(I, T_{X(0)}^{*} M\right):\right. \\
& \left.\quad \exists b \in \Omega^{0}\left(I, T_{X(0)}^{*} M\right), \quad b(0) \in N_{0}^{*}, U(1)^{t} b(1) \in N_{1}^{*}, \tilde{\lambda}=\pi_{0}^{\sharp} b, \widetilde{\phi}=-\mathrm{d} b\right\} .
\end{aligned}
$$

Observe that from this description of $T_{(X, \eta)}^{\perp} \mathcal{C}_{\pi}\left(M ; C_{0}, C_{1}\right)$ it follows immediately that

$$
T_{(X, \eta)}^{\perp \perp} \mathcal{C}_{\pi}\left(M ; C_{0}, C_{1}\right)=T_{(X, \eta)} \mathcal{C}_{\pi}\left(M ; C_{0}, C_{1}\right) .
$$

If we now invert the transformations (2.10a) and (2.10b) to go back to tangent vectors at $(X, \eta)$,

$$
\begin{aligned}
& \widetilde{\xi}=U^{-1} \widetilde{\lambda} \in \Gamma\left(X^{*} T M\right), \\
& \widetilde{e}=U^{t} \widetilde{\phi} \in \Gamma\left(T^{*} I \otimes X^{*} T^{*} M\right),
\end{aligned}
$$

and introduce

$$
\beta=U^{t} b \in \Gamma\left(X^{*} T^{*} M\right),
$$

we obtain that the characteristic distribution of the coisotropic submanifold $\mathcal{C}_{\pi}\left(M ; C_{0}, C_{1}\right)$ is given, at the point $(X, \eta)$, by the family of vectors $(\widetilde{\xi}, \widetilde{e}) \in T_{(X, \eta)}$ $\mathcal{C}_{\pi}\left(M ; C_{0}, C_{1}\right)$ defined by 


$$
\begin{aligned}
& \widetilde{\xi}=\pi^{\sharp}(X) \beta, \\
& \widetilde{e}=-D \beta,
\end{aligned}
$$

for $\beta \in \Gamma\left(X^{*} T^{*} M\right)$ with $\beta(0) \in N_{X(0)}^{*} C_{0}$ and $\beta(1) \in N_{X(1)}^{*} C_{1}$. In local coordinates the above formulae read,

$$
\begin{aligned}
& \widetilde{\xi}^{i}=-\pi^{i j}(X) \beta_{j}, \\
& \widetilde{e}_{i}=-\mathrm{d} \beta_{i}+\Gamma_{r i}^{k}(X) \mathrm{d} X^{r} \beta_{k}+(\nabla \pi)_{i}^{j k}(X) \eta_{j} \beta_{k} .
\end{aligned}
$$

These are the symmetries of the Poisson sigma model as presented in $[6,11,13]$. The boundary conditions for $\beta$ in case of coisotropic boundary conditions has been introduced in [7].

\subsection{2. "Equivariant Momentum Map"}

Denote by $\iota_{C}$ the inclusion map of a submanifold $C$ into a manifold $M$ and define

$$
\Omega_{C}^{1}(M)=\left\{\alpha \in \Omega^{1}(M): \iota_{C}^{*} \alpha=0\right\} .
$$

LEMMA 3.7. If $M$ is a Poisson manifold and $C$ is a coisotropic submanifold, then $\Omega_{C}^{1}(M)$ is a Lie subalgebra of $\Omega^{1}(M)$.

We leave the proof of this simple fact (which directly follows from $N^{*} C$ being a Lie subalgebroid of $T^{*} M$ ) to the reader.

Now, given two coisotropic submanifolds $C_{0}$ and $C_{1}$, we define the Lie algebra

$$
P_{C_{0}, C_{1}} \Omega^{1}(M)=\left\{B: I \rightarrow \Omega^{1}(M): B(i) \in \Omega_{C_{i}}^{1}(M), i=0,1\right\},
$$

where the Lie bracket is defined pointwise. The map $B$ is assumed to be continuously differentiable. Given a path $X$ on $M$ and an element $B$ of this Lie algebra, we define $B_{X} \in \Gamma\left(X^{*} T^{*} M\right)$ by

$$
B_{X}(u)=B(u)(X(u)) .
$$

If $X(i) \in C_{i}, i=0,1$, then $B_{X}(i) \in N_{X(i)}^{*} M$. It is not difficult to check that, replacing $\beta$ by $B_{X}$ in (3.15), one may define an infinitesimal action of $P_{C_{0}, C_{1}} \Omega^{1}(M)$ on $\mathcal{C}_{\pi}\left(M ; C_{0}, C_{1}\right)$ whose induced foliation is the canonical foliation. On $T^{*} P M\left(C_{0}, C_{1}\right)$ one may also define Hamiltonian functions for this action; viz.,

$$
\mu_{B}(X, \eta):=\int_{I}\left\langle B_{X}, \mathrm{~d} X+\pi^{\sharp}(X) \eta\right\rangle .
$$

\subsection{PROOF OF THEOREM 1.5}

We proceed as in Section 2.1. Proposition 2.1 still holds, so we have 


$$
T_{(X, \eta)} \mathcal{C}_{\pi}(M)=\left\{\xi \in \Gamma\left(X^{*} T M\right), e \in \Gamma\left(T^{*} S^{1} \otimes X^{*} T^{*} M\right):(D \xi)+\pi^{\sharp}(X) e=0\right\} .
$$

We now regard $S^{1}$ as the interval $I=[0,1]$ with identified end points. The fields are then regarded as periodic sections on it. We then continue likewise up to (2.10) getting

$$
\begin{aligned}
& T_{(X, \eta)} \mathcal{C}_{\pi}(M) \cong T_{(X, \eta)} \mathcal{C}_{\pi}(M)^{\mathrm{twisted}} \\
& :=\left\{\lambda \in \Omega^{0}\left(I, T_{X(0)} M\right), \phi \in \Omega^{1}\left(I, T_{X(0)}^{*} M\right): \mathrm{d} \lambda+P^{\sharp} \phi=0,\right. \\
& \left.\quad \lambda(1)=U(1) \lambda(0), \phi(0)=U(1)^{t} \phi(1)\right\}
\end{aligned}
$$

and

$$
\begin{aligned}
T_{(X, \eta)} \mathcal{C}_{\pi}(M) \cong T_{(X, \eta)} \mathcal{C}_{\pi}(M)_{0}:= & \left\{\lambda_{0} \in T_{X(0)} M, \phi \in \Omega^{1}\left(I, T_{X(0)}^{*} M\right):\right. \\
& \left.U(1) \lambda_{0}=\lambda_{0}-\int_{I} P^{\sharp} \phi, \phi(0)=U(1)^{t} \phi(1)\right\} .
\end{aligned}
$$

We then proceed as in Section 2.2 getting

$$
\begin{aligned}
T_{(X, \eta)}^{\perp} \mathcal{C}_{\pi}(M) \cong T_{(X, \eta)}^{\perp} \mathcal{C}_{\pi}(M)^{\text {implicit }} \\
:=\left\{\tilde{\lambda} \in \Omega^{0}\left(I, T_{X(0)} M\right), \widetilde{\phi} \in \Omega^{1}\left(I, T_{X(0)}^{*} M\right): \quad \tilde{\lambda}(1)=U(1) \widetilde{\lambda}(0), \widetilde{\phi}(0)=U(1)^{t} \widetilde{\phi}(1),\right. \\
\left.\quad \int_{I}\left\langle\phi, \tilde{\lambda}+\left(P^{\sharp}\right)^{t} \int_{\bullet}^{1} \widetilde{\phi}\right\rangle-\left\langle\int_{I} \widetilde{\phi}, \lambda_{0}\right\rangle=0, \quad \forall\left(\lambda_{0}, \phi\right) \in T_{(X, \eta)} \mathcal{C}_{\pi}(M)_{0}\right\} .
\end{aligned}
$$

PROPOSITION 3.8. If $\pi$ is a Poisson bivector field, then $\mathcal{C}_{\pi}(M)$ is coisotropic.

Proof. By Lemma 2.2 we have $P=\pi_{0}$, so the condition on $\lambda_{0}$ becomes $U(1) \lambda_{0}=$ $\lambda_{0}-\pi_{0}^{\sharp} \int_{I} \phi$, which is in particular satisfied by $\lambda_{0}=0$ assuming $\int_{I} \phi=0$. In particular, we get

$$
\int_{I}\left\langle\phi, \tilde{\lambda}-\pi_{0}^{\#} \int_{\bullet}^{1} \tilde{\phi}\right\rangle=0,
$$

for all $\phi \in \Omega^{1}\left(I, T_{X(0)}^{*} M\right)$ satisfying $\phi(0)=U(1)^{t} \phi(1)$ and $\int_{I} \phi=0$. This implies $\mathrm{d}\left(\widetilde{\lambda}-\pi_{0}^{\#} \int_{\bullet}^{1} \widetilde{\phi}\right)=0$, so $(\widetilde{\lambda}, \widetilde{\phi}) \in T_{(X, \eta)} \mathcal{C}_{\pi}(M)_{0}$.

PROPOSITION 3.9. If $\mathcal{C}_{\pi}(M)$ is coisotropic, then $\pi$ is a Poisson bivector field.

Proof. Notice that any $(\widetilde{\lambda}, \widetilde{\phi})$ with $\widetilde{\phi}(0)=U(1)^{t} \widetilde{\phi}(1)$ satisfying (3.1) and (3.2) belongs to $T_{(X, \eta)}^{\perp} \mathcal{C}_{\pi}(M)^{\text {implicit }}$ and hence, since $\mathcal{C}_{\pi}(M)$ is coisotropic, to $T_{(X, \eta)}$ $\mathcal{C}_{\pi}(M)^{\text {twisted }}$. The proof then proceeds exactly as in the proof to Proposition 3.2.

This concludes the proof of Theorem 1.5. 


\subsubsection{Symmetries}

Assume $\pi$ is Poisson. In the proof to Proposition 3.8 we have only considered $\lambda_{0}=$ 0 and $\int_{I} \phi=0$, which yields only some necessary condition to be satisfied by $(\widetilde{\lambda}, \widetilde{\phi})$. We now want to characterize $T^{\perp} \mathcal{C}_{\pi}(M)$ completely.

PROPOSITION 3.10. The characteristic distribution of $\mathcal{C}_{\pi}(M)$ at $(X, \eta)$ is given by the family of vectors $(\widetilde{\xi}, \widetilde{e}) \in T_{(X, \eta)} \mathcal{C}_{\pi}(M)$ defined by

$$
\begin{aligned}
& \widetilde{\xi}=\pi^{\sharp}(X) \beta, \\
& \tilde{e}=-D \beta,
\end{aligned}
$$

with $\beta \in \Gamma\left(X^{*} T^{*} M\right)$.

Proof. We have already obtained that if $(\widetilde{\lambda}, \widetilde{\phi})$ belongs to $T^{\perp} \mathcal{C}_{\pi}(M)^{\text {implicit }}$ then, in addition to $\widetilde{\lambda}(1)=U(1) \widetilde{\lambda}(0)$ and $\widetilde{\phi}(0)=U(1)^{t} \widetilde{\phi}(1)$, it satisfies $\mathrm{d}\left(\widetilde{\lambda}-\pi_{0}^{\sharp} \int_{0}^{1} \widetilde{\phi}\right)=0$. This implies that $(\widetilde{\lambda}, \widetilde{\phi})$ belongs to $T^{\perp} \mathcal{C}_{\pi}(M)^{\text {implicit }}$ iff in addition

$$
\left\langle\int_{I} \phi, \tilde{\lambda}(1)\right\rangle-\left\langle\int_{I} \tilde{\phi}, \lambda_{0}\right\rangle=0
$$

for all $\left(\lambda_{0}, \phi\right) \in T_{(X, \eta)} \mathcal{C}_{\pi}(M)_{0}$.

First consider $\lambda_{0}=0$ and $\int_{I} \phi \in \operatorname{ker} \pi_{0}^{\sharp}$. This yields, by Lemma 3.4, that there is a $\theta \in T_{X(0)}^{*} M$ with $\widetilde{\lambda}(1)=\pi_{0}^{\sharp}(\theta)$; hence

$$
\tilde{\lambda}(u)=\pi_{0}^{\sharp}\left(\theta+\int_{u}^{1} \tilde{\phi}\right) .
$$

As a consequence $\left\langle\int_{I} \phi, \tilde{\lambda}(1)\right\rangle=0$, so we are left with the condition $\left\langle\int_{I} \widetilde{\phi}, \lambda_{0}\right\rangle=0$ for all $\lambda_{0}$ such that there is a $\phi$ with $\left(\lambda_{0}, \phi\right) \in T_{(X, \eta)} \mathcal{C}_{\pi}(M)_{0}$. In particular, we may take $\phi$ such that $\int_{I} \phi$ is in the kernel of $\pi_{0}^{\sharp}$; since $\pi_{0}^{\sharp} \int_{I} \phi=\lambda_{0}-U(1) \lambda_{0}$, this yields that $\lambda_{0}$ must now lie in the kernel of the operator $G:=U(1)-$ id. Since $\left\langle\int_{I} \widetilde{\phi}, \lambda_{0}\right\rangle$ must vanish for all $\lambda_{0}$ satisfying this condition, we get that $\int_{I} \widetilde{\phi}$ must be in the image of $G^{t}$. Hence there is a $\gamma \in T_{X(0)}^{*} M$ with

$$
\int_{I} \widetilde{\phi}=U(1)^{t} \gamma-\gamma
$$

Using again $\pi_{0}^{\sharp} \int_{I} \phi=\lambda_{0}-U(1) \lambda_{0}$, we now get $\left\langle\int_{I} \widetilde{\phi}, \lambda_{0}\right\rangle=\left\langle\int_{I} \phi, \pi_{0}^{\sharp} \gamma\right\rangle$; so condition (3.18) finally reads $\left\langle\int_{I} \phi, \tilde{\lambda}(1)-\pi_{0}^{\sharp} \gamma\right\rangle=0$ for all $\phi$ such that there is a $\lambda_{0}$ with $\left(\lambda_{0}, \phi\right) \in T_{(X, \eta)} \mathcal{C}_{\pi}(M)_{0}$. Using (3.19), the condition becomes $\left\langle\theta-\gamma, \pi_{0}^{\sharp} \int_{I} \phi\right\rangle=$ 0 or, using $\pi_{0}^{\sharp} \int_{I} \phi=-G \lambda_{0},\left\langle\theta-\gamma, G \lambda_{0}\right\rangle=0$. Hence we have that $\theta-\gamma$ must be in the annihilator of $\operatorname{im} \pi_{0}^{\sharp} \cap \operatorname{im} G$ which is $\operatorname{ker} \pi_{0}^{\sharp}+\operatorname{ker} G^{t}$. We hence have $\mu, v \in$ $T_{X(0)}^{*} M$ with $\pi_{0}^{\sharp} \mu=0$ and $U(1)^{t} v=v$ such that $\theta-\gamma=\mu+v$. Finally, define 


$$
b(u):=\theta-\mu+\int_{u}^{1} \widetilde{\phi}=v+\gamma+\int_{u}^{1} \widetilde{\phi} .
$$

Since $U(1)^{t} v=v$, we have thanks to (3.20) that $b(0)=U(1)^{t} b(1)$. This shows that

$$
\beta:=U^{t} b
$$

is a periodic section of $X^{*} T^{*} M$. Since $\pi_{0}^{\sharp} \mu=0$, we have from (3.19) that $\widetilde{\lambda}=\pi_{0}^{\sharp} b$. Moreover, since $\theta$ and $\mu$ are constant, we have $\widetilde{\phi}=-\mathrm{d} b$.

If we now invert the transformations (2.10a) and (2.10b) to go back to tangent vectors at $(X, \eta)$

$$
\begin{aligned}
& \widetilde{\xi}=U^{-1} \widetilde{\lambda} \in \Gamma\left(X^{*} T M\right), \\
& \widetilde{e}=U^{t} \widetilde{\phi} \in \Gamma\left(T^{*} S^{1} \otimes X^{*} T^{*} M\right),
\end{aligned}
$$

we obtain that a pair $(\widetilde{\xi}, \widetilde{e})$ belongs to $T^{\perp} \mathcal{C}_{\pi}(M)$ iff equations $(3.17 \mathrm{a})$ and $(3.17 \mathrm{~b})$ are satisfied.

Remark 3.11. One may define functions $\mu_{B}$ on $T^{*} L M$ as in (3.16) with $B$ now a map from $S^{1}$ to $\Omega^{1}(M)$. Notice that the functions $\mu_{B}$ generate the vanishing ideal of $\mathcal{C}_{\pi}(M)$ and that their Hamiltonian vector fields generate the distribution defined by (3.17a) and (3.17b). This remark however does not replace the proof above as in the infinite dimensional case it is not automatic that the Hamiltonian vector fields of functions in the vanishing ideal span the whole characteristic distribution.

\section{Dual Pairs}

In this section we assume that $C_{0}$ and $C_{1}$ are coisotropic submanifolds of a Poisson manifold $M$. In this case $\mathcal{C}_{\pi}\left(M ; C_{0}, C_{1}\right)$ is a coisotropic submanifold of $T^{*} P M$ and its leaf space $\mathcal{C}_{\pi}\left(M ; C_{0}, C_{1}\right)$ is endowed with a symplectic structure. On the other hand, the leaf spaces $C_{0}$ and $C_{1}$ are endowed with a Poisson structure. By (3.15a) and the conditions on $\beta$, the maps $p_{i}$ of (1.1) descend to the quotients

$$
\underline{p}_{i}: \underline{\mathcal{C}_{\pi}\left(M ; C_{0}, C_{1}\right)} \rightarrow \underline{C_{i}}, \quad i=0,1 .
$$

Proceeding as in the proof of Theorem 4.6 in [6], one may prove that $\underline{p}_{0}$ and $\underline{p}_{1}$ are a Poisson and an anti-Poisson map respectively. We will prove the following

LEMMA 4.1. $\operatorname{kerd} \underline{p}_{0}$ and $\operatorname{kerd} \underline{p}_{1}$ are symplectically orthogonal at any point of $\mathcal{C}_{\pi}\left(M ; C_{0}, C_{1}\right)$, i.e.,

$$
\left(\operatorname{kerd} \underline{p}_{0}(x)\right)^{\perp}=\operatorname{kerd} \underline{p}_{1}(x), \quad \forall x \in \underline{\mathcal{C}_{\pi}\left(M ; C_{0}, C_{1}\right)} .
$$

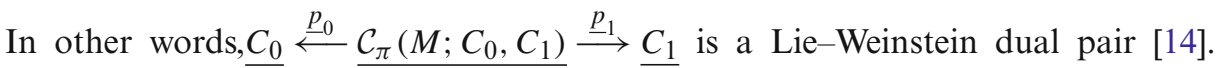
Observe that the maps $\underline{p}_{i}$ may fail to be surjective submersions, so this dual pair is in general not full. 
Remark 4.2. Since the quotient $\mathcal{C}_{\pi}\left(M ; C_{0}, C_{1}\right)$ is finite dimensional, the above condition is equivalent to

$$
\left(\operatorname{kerd} \underline{p}_{1}(x)\right)^{\perp}=\operatorname{kerd} \underline{p}_{0}(x), \quad \forall x \in \underline{\mathcal{C}}_{\pi}\left(M ; C_{0}, C_{1}\right) .
$$

Notice that the maps $\underline{p}_{i} \mathrm{~s}$ are defined, as continuous maps, even if the leaf spaces are not smooth. Lemma 4.1 makes sense also in the nonsmooth case if we

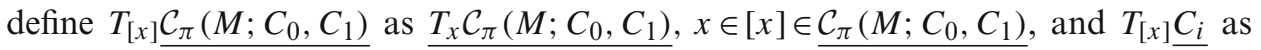
$\underline{T_{x} C_{i}}, x \in[x] \in \underline{C_{i}}$. The linear maps kerd $\underline{p}_{0}$ and ker $\underline{p}_{1}$ are also well defined. Thus, we may think of $C_{0} \stackrel{\underline{p}_{0}}{\longleftarrow} \underline{\mathcal{C}}_{\pi}\left(M ; C_{0}, C_{1}\right) \stackrel{\underline{p}_{1}}{\longrightarrow} \underline{C_{1}}$

as of a singular Lie-Weinstein dual pair.

Proof of Lemma 4.1. Let $(X, \eta)$ be a representative of $x \in \underline{\mathcal{C}_{\pi}\left(M ; C_{0}, C_{1}\right)}$. We introduce the following notations:

$$
\begin{aligned}
V & :=T_{(X, \eta)} \mathcal{C}_{\pi}\left(M ; C_{0}, C_{1}\right), \\
Z_{i} & :=T_{X(i)} C_{i}, \\
Z_{i}^{\perp} & :=\pi^{\sharp}(X(i))\left(N_{X(i)}^{*} C_{i}\right), \\
\varpi_{i} & :=\mathrm{d} p_{i}(X, \eta),
\end{aligned}
$$

for $i=0,1$. So we have the following commutative diagram of vector spaces:

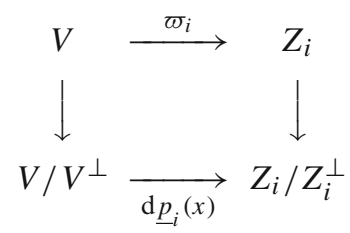

We then have

$$
\begin{aligned}
\operatorname{kerd} \underline{p}_{i}(x) & =\varpi_{i}^{-1}\left(Z_{i}^{\perp}\right) / V^{\perp}, \\
\left(\operatorname{kerd} \underline{p}_{i}(x)\right)^{\perp} & =\left(\varpi_{i}^{-1}\left(Z_{i}^{\perp}\right)\right)^{\perp} / V^{\perp},
\end{aligned}
$$

and

$$
\begin{array}{r}
\varpi_{0}^{-1}\left(Z_{0}^{\perp}\right)=\left\{\lambda \in \Omega^{0}\left(I, T_{X(0)} M\right), \quad \phi \in \Omega^{1}\left(I, T_{X(0)}^{*} M\right): \mathrm{d} \lambda+\pi_{0}^{\sharp} \phi=0,\right. \\
\left.\lambda(0) \in \pi_{0}^{\sharp}\left(N_{0}^{*}\right), U(1)^{-1} \lambda(1) \in T_{1}\right\}, \\
\varpi_{1}^{-1}\left(Z_{1}^{\perp}\right)=\left\{\lambda \in \Omega^{0}\left(I, T_{X(0)} M\right), \quad \phi \in \Omega^{1}\left(I, T_{X(0)}^{*} M\right): \mathrm{d} \lambda+\pi_{0}^{\sharp} \phi=0,\right. \\
\left.\lambda(0) \in T_{0}, U(1)^{-1} \lambda(1) \in \pi_{1}^{\sharp}\left(N_{1}^{*}\right)\right\} .
\end{array}
$$

Step 1: $\left(\operatorname{ker} \mathrm{d} \underline{p}_{1}(x)\right) \subset \operatorname{kerd} \underline{p}_{0}(x)^{\perp}$.

It is enough to show that

$$
\Omega_{(X, \eta)}((\xi, \zeta),(\widetilde{\xi}, \widetilde{\zeta}))=0, \quad \forall(\xi, \zeta) \in \varpi_{0}^{-1}\left(Z_{0}^{\perp}\right), \quad(\widetilde{\xi}, \widetilde{\zeta}) \in \varpi_{1}^{-1}\left(Z_{1}^{\perp}\right),
$$


since this implies $\varpi_{1}^{-1}\left(Z_{1}^{\perp}\right)^{\perp} \subset \varpi_{0}^{-1}\left(Z_{0}^{\perp}\right)^{\perp}$ which in turn implies the desired result on the quotient. Using (2.13) with

$$
\begin{aligned}
& \lambda=\lambda_{0}-\pi_{0}^{\sharp} \int_{0}^{\bullet} \phi, \\
& \tilde{\lambda}=\widetilde{\lambda}_{1}+\pi_{0}^{\sharp} \int_{\bullet}^{1} \widetilde{\phi},
\end{aligned}
$$

we get

$$
\Omega_{(X, \eta)}((\xi, \zeta),(\widetilde{\xi}, \widetilde{\zeta}))=\left\langle\int_{I} \phi, \widetilde{\lambda}_{1}\right\rangle-\left\langle\int_{I} \widetilde{\phi}, \lambda_{0}\right\rangle .
$$

If we now write

$$
\begin{aligned}
& \lambda_{0}=\pi_{0}^{\sharp} \alpha, \\
& \tilde{\lambda}_{1}=U(1) \pi_{1}^{\sharp} \beta=\pi_{0}^{\sharp}\left(U^{t}\right)^{-1} \beta,
\end{aligned}
$$

with $\alpha \in N_{0}^{*}$ and $\beta \in N_{1}^{*}$, we get

$$
\left\langle\int_{I} \phi, \tilde{\lambda}_{1}\right\rangle=\left\langle\int_{I} \widetilde{\phi}, \lambda_{0}\right\rangle=-\left\langle U(1)^{-1} \pi_{0}^{\sharp} \alpha, \beta\right\rangle,
$$

which completes Step 1.

Step 2: $\left(\operatorname{ker} \mathrm{d} \underline{p}_{0}(x)\right)^{\perp} \subset \operatorname{ker} \mathrm{d} \underline{p}_{1}(x)$.

Let $(\widetilde{\lambda}, \widetilde{\phi})$ be an element of $\left(\varpi_{0}^{-1}\left(Z_{0}^{\perp}\right)\right)^{\perp}$. Then proceeding exactly as in the proof of Proposition 3.3, we see that this element must satisfy (3.6) with $\tilde{\lambda}_{1}=$ $U(1) \pi_{1}^{\sharp} \theta$ for some $\theta \in N_{1}^{*}$. We still have condition (3.10) but now for all $\lambda_{0} \in$ $\pi_{0}^{\sharp}\left(N_{0}^{*}\right)$. This implies that

$$
\left(U^{t}\right)^{-1} \theta+\int_{I} \widetilde{\phi} \in \operatorname{Ann}\left(\pi_{0}^{\sharp} N_{0}^{*}\right) .
$$

In finite dimensions, Lemma 3.4 on page 12 implies $F^{-1}(T)=\operatorname{Ann}\left(F^{t}(\operatorname{Ann}(T))\right)$. Taking $T=T_{0}$ and $F=\pi_{0}^{\sharp}$, we then get

$$
\operatorname{Ann}\left(\pi_{0}^{\sharp} N_{0}^{*}\right)=\operatorname{Ann}\left(\pi_{0}^{\sharp} \operatorname{Ann}\left(T_{0}\right)\right)=\left(\pi_{0}^{\sharp}\right)^{-1}\left(T_{0}\right) .
$$

Thus, (3.13) implies

$$
\tilde{\lambda}(0)=\pi_{0}^{\sharp}\left(\left(U^{t}\right)^{-1} \theta+\int_{I} \tilde{\phi}\right) \in T_{0} .
$$

Hence $(\widetilde{\lambda}, \widetilde{\phi})$ is an element of $\varpi_{1}^{-1}\left(Z_{1}^{\perp}\right)$, and its class modulo $V^{\perp}$ is an element of $\operatorname{kerd} \underline{p}_{1}(x)$. 


\subsection{COMPOSITION}

Under certain technical conditions (see [12] and references therein), dual pairs can be composed by symplectic reduction. Namely, let $P_{0}, P_{1}$ and $P_{2}$ be Poisson manifolds, $S_{0}$ and $S_{1}$ symplectic manifolds, together with Poisson maps $I_{0}, J_{0}$, and antiPoisson maps $I_{1}$ and $J_{1}$ as in the following diagram:

$$
P_{0} \stackrel{I_{0}}{\longleftarrow} S_{0} \stackrel{I_{1}}{\longrightarrow} P_{1} \stackrel{J_{0}}{\longleftarrow} S_{1} \stackrel{J_{1}}{\longrightarrow} P_{2},
$$

then $S_{0} \times{ }_{P_{1}} S_{1}$ is a coisotropic submanifold of $S_{0} \times \overline{S_{1}}\left(\overline{S_{1}}\right.$ denotes $S_{1}$ with opposite symplectic structure), and the maps $I_{0}, J_{1}$ descend to the symplectic quotients, so that

$$
P_{0} \stackrel{I_{0}}{\longleftarrow} \underline{S_{0} \times P_{1} S_{1}} \stackrel{\underline{J_{1}}}{\longrightarrow} P_{2}
$$

is a new dual pair which we will denote by

$$
S_{0} \star S_{1}
$$

Of course, without the appropriate assumptions, this might be quite singular; even if we started with smooth manifolds, already the fibered product $S_{0} \times{ }_{P_{1}} S_{1}$ might not be a manifold, unless $I_{1}$ and $J_{0}$ are surjective submersions. For the reduced space to be smooth as well, one need some more assumptions, see [12].

In our case, we allow all sorts of singularity. Given coisotropic submanifolds $C_{0}, C_{1}$ and $C_{2}$, we can construct singular dual pairs by $\mathcal{C}_{\pi}\left(M ; C_{0}, C_{1}\right)$ and

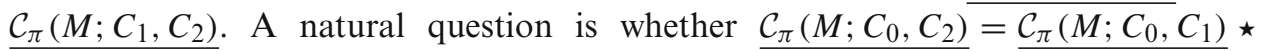
$\overline{\mathcal{C}_{\pi}\left(M ; C_{1}, C_{2}\right)}$. Roughly speaking the composition of these dual pairs arises by joining paths at a fiber of $C_{1} \rightarrow C_{1}$. So we may expect the above identity to hold only if every path from $C_{0}$ to $C_{2}$ is equivalent to a path that passes through $C_{1}$. Otherwise in the composition we will select only paths with this property, so we may expect that, in general, only the following inclusion relation holds:

$$
\underline{\mathcal{C}_{\pi}\left(M ; C_{0}, C_{2}\right)} \supset \underline{\mathcal{C}_{\pi}\left(M ; C_{0}, C_{1}\right)} \star \underline{\mathcal{C}_{\pi}\left(M ; C_{1}, C_{2}\right)} .
$$

In the next section, through another description of our singular dual pairs, this will be more clear. Another way out is the extension to this case of the construction in $[3,9]$, where we might speak of relational dual pairs.

\section{Reduced Spaces}

Let $(M, \pi)$ be a Poisson manifold, then $\mathrm{G}(M)=\mathcal{C}_{\pi}(M ; M, M)$ is the (possibly singular) source-simply-connected symplectic groupoid of $M$ [6]. In this case we will denote by $s$ and $t$ (instead of $\underline{p}_{0}$ and $\underline{p}_{1}$ ) the Poisson and anti-Poisson maps to $M$. Given two submanifolds $C_{0}$ and $C_{1}$ of $M$, we define

$$
\mathcal{C}_{\pi}^{M ; C_{0}, C_{1}}=s^{-1}\left(C_{0}\right) \cap t^{-1}\left(C_{1}\right) .
$$

If $C_{0}$ and $C_{1}$ are coisotropic, then so are $s^{-1}\left(C_{0}\right), t^{-1}\left(C_{1}\right)$ and (because of the symplectic orthogonality of the $s$ - and $t$-fibers) also $\mathcal{C}_{\pi}^{M ; C_{0}, C_{1}}$. We may then consider its reduction $\underline{\mathcal{C}_{\pi}^{M ; C_{0}}, C_{1}}$. We have the following 
THEOREM 5.1. $\underline{\mathcal{C}_{\pi}^{M ; C_{0}, C_{1}}}=\underline{\mathcal{C}_{\pi}\left(M ; C_{0}, C_{1}\right)}$.

This Theorem is a consequence of the following

LEMMA 5.2 (Reduction in stages). Let $S$ be a (possibly infinite-dimensional, weak) symplectic space. Let $V$ be a subspace of $S$ and $W$ a subspace of $V$. If $W$ is coisotropic in $S$, then:

(1) $V^{\perp} \subset W^{\perp} \subset W \subset V$, and in particular $V$ is also coisotropic.

(2) $W / V^{\perp}$ is coisotropic in $V / V^{\perp}$.

(3) $W / W^{\perp}=\left(W / V^{\perp}\right) /\left(W / V^{\perp}\right)^{\perp}$.

The proof is a simple exercise in linear algebra.

Proof of Theorem 5.1. Let $x$ be a point in $\mathcal{C}_{\pi}^{M ; C_{0}, C_{1}} \subset \mathrm{G}(M)$ and $(X, \eta)$ a representative of $x$ in $\mathcal{C}_{\pi}\left(M ; C_{0}, C_{1}\right) \subset \mathcal{C}_{\pi}(M ; M, M)$. Then we apply the reduction in stages to the following spaces

$$
\begin{aligned}
S & :=T_{(X, \eta)} T^{*} P M, \\
V & :=T_{(X, \eta)} \mathcal{C}_{\pi}(M ; M, M), \\
W & :=T_{(X, \eta)} \mathcal{C}_{\pi}\left(M ; C_{0}, C_{1}\right),
\end{aligned}
$$

observing that

$$
W / V^{\perp}=T_{x} \mathcal{C}_{\pi}^{M ; C_{0}, C_{1}} .
$$

Thanks to Theorem 5.1 we may now easily discuss a few examples.

EXAMPLE 5.3 (Trivial Poisson structure). For $\pi=0$ we have $\mathrm{G}(M)=T^{*} M$ with canonical symplectic structure and with $s=t=$ projection $T^{*} M \rightarrow M$. Any submanifold of $M$ is automatically coisotropic with trivial foliation. Then we have

$$
\mathcal{C}_{0}\left(M ; C_{0}, C_{1}\right)=T_{C_{0} \cap C_{1}}^{*} M
$$

and

$$
\underline{\mathcal{C}_{0}\left(M ; C_{0}, C_{1}\right)}=T^{*}\left(C_{0} \cap C_{1}\right)
$$

which is a manifold iff $C_{0} \cap C_{1}$ is so. Moreover, a simple computation shows that

$$
\underline{\mathcal{C}_{0}\left(M ; C_{0}, C_{1}\right)} \star \underline{\mathcal{C}_{0}\left(M ; C_{1}, C_{2}\right)}=T^{*}\left(C_{0} \cap C_{1} \cap C_{2}\right) \subset \underline{\mathcal{C}_{0}\left(M ; C_{0}, C_{2}\right)} .
$$


EXAMPLE 5.4 (Symplectic case). Let $M$ be a symplectic manifold, and $\pi$ the corresponding Poisson structure. For simplicity we assume $M$ to be simply connected. Then $\mathrm{G}(M)=M \times \bar{M}$, where $\bar{M}$ denotes $M$ with opposite symplectic structure. The maps $s$ and $t$ are the projections to the factors. Thus,

$$
\mathcal{C}_{\pi}\left(M ; C_{0}, C_{1}\right)=C_{0} \times C_{1},
$$

and, in case $C_{0}$ and $C_{1}$ are coisotropic,

$$
\underline{\mathcal{C}_{\pi}\left(M ; C_{0}, C_{1}\right)}=\underline{C_{0}} \times \underline{C_{1}} .
$$

In this case,

$$
\underline{\mathcal{C}_{\pi}\left(M ; C_{0}, C_{2}\right)}=\underline{\mathcal{C}_{\pi}\left(M ; C_{0}, C_{1}\right)} \star \underline{\mathcal{C}_{\pi}\left(M ; C_{1}, C_{2}\right)},
$$

for any three coisotropic submanifolds $C_{0}, C_{1}$ and $C_{2}$.

Observe that the map to $M$ from $\mathcal{C}_{\pi}^{M ; C_{0}, M}$ (resp., $\mathcal{C}_{\pi}^{M ; M, C_{1}}$ ) is a surjective submersion if $C_{0}$ (resp., $C_{1}$ ) has a clean intersection with every symplectic leaf of $M$. Under this condition, the dual pairs behave well and the composition is well defined (in the world of differentiable stacks). Thus, if we define a coisotropic submanifold to be nice when it has a clean intersection with every symplectic leaf, we have the following

THEOREM 5.5. To every Poisson manifold $(M, \pi)$, we may associate a category $\mathrm{C}(M)$ where the objects are Poisson reductions (as differentiable stacks) of nice coisotropic submanifolds of $M$ and the morphisms from the object $C_{0}$ to the object

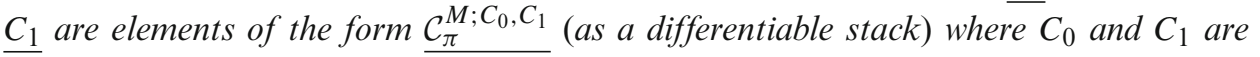
nice coisotropic submanifolds of $M$ which reduce to $\underline{C_{0}}$ and $\underline{C_{1}}$.

\subsection{GROUPOID QUOTIENTS}

As shown in [2], there is a one-to-one correspondence between coisotropic submanifolds of a given Poisson manifold $M$ and (possibly singular) Lagrangian subgroupoids of the (possibly singular) source-simply-connected symplectic groupoid $\mathrm{G}(M)$ of $M$. It turns out that $\mathcal{C}_{\pi}^{M ; C_{0}, C_{1}}$ may be understood as a quotient of groupoids. Namely, given a groupoid $\mathrm{G} \rightrightarrows M$ and a subgroupoid $\mathrm{L} \rightrightarrows C \subset M$, we have a left action of $\mathrm{L}$ on $s^{-1}(C)$ and a right action on $t^{-1}(C)$. We will write $\mathrm{L} \backslash \mathrm{G}$ (resp., G/L) as a shorthand notation for $\mathrm{L} \backslash s^{-1}(C)$ (resp., $t^{-1}(C) / \mathrm{L}$ ). If $\mathrm{L}(C)$ is the Lagrangian subgroupoid of $\mathrm{G}(M)$ corresponding to the coisotropic submanifold $C$, we have

$$
\begin{aligned}
& \mathcal{C}_{\pi}(M ; C, M)=\mathrm{L}(C) \backslash \mathrm{G}(M), \\
& \underline{\mathcal{C}_{\pi}(M ; M, C)}=\mathrm{G}(M) / \mathrm{L}(C) .
\end{aligned}
$$


For two given coisotropic submanifolds $C_{0}$ and $C_{1}$, we have instead

$$
\begin{aligned}
\underline{\mathcal{C}_{\pi}\left(M ; C_{0}, C_{1}\right)} & =\mathrm{L}\left(C_{0}\right) \backslash \mathrm{G}(M) / \mathrm{L}\left(C_{1}\right) \\
& =\mathrm{L}\left(C_{0}\right) \backslash\left(s^{-1}\left(C_{0}\right) \cap t^{-1}\left(C_{1}\right)\right) / \mathrm{L}\left(C_{1}\right) .
\end{aligned}
$$

This can be verified by recalling the construction of [2] and comparing it with the one in this paper. Namely, the results of Section 3.2.1, may be rephrased as follows:

$$
\begin{array}{r}
\mathcal{C}_{\pi}\left(M ; C_{0}, C_{1}\right)=\left\{\text { Lie algebroid morphisms } T I \rightarrow T^{*} M\right. \\
\text { with base maps connecting } \left.C_{0} \text { to } C_{1}\right\} .
\end{array}
$$

Two elements $\gamma_{0}$ and $\gamma_{1}$ of $\mathcal{C}_{\pi}\left(M ; C_{0}, C_{1}\right)$ are defined to be equivalent if there exists a Lie algebroid morphism $\Gamma: T(I \times J) \rightarrow T^{*} M$, with $J$ an interval, such that

(1) the restriction of $\Gamma$ to the boundaries of $J$ are the two given morphisms $\gamma_{0}$ and $\gamma_{1}$, and

(2) the restriction of $\Gamma$ to the boundaries $\{0\}$ and $\{1\}$ of $I$ are Lie algebroid morphism $T J \rightarrow N^{*} C_{i}, i=0,1$.

Then $\mathcal{C}_{\pi}\left(M ; C_{0}, C_{1}\right)$ may be regarded as the quotient of $\mathcal{C}_{\pi}\left(M ; C_{0}, C_{1}\right)$ by this equivalence relation.

On the other hand, the Lagrangian subgroupoid corresponding to a coisotropic submanifold $C$ is shown in [2] to be the source-simply-connected groupoid whose Lie algebroid is $N^{*} C$, and this is exactly [10] the quotient of the space of Lie algebroid morphisms $T J \rightarrow N^{*} C$ by Lie algebroid morphisms $T(J \times K) \rightarrow N^{*} C$ (where $K$ is another interval) which are trivial on the boundary of $J$. Finally observe that equivalent Lie algebroid morphisms $T J \rightarrow N^{*} C_{i}$ act the same way on $\mathcal{C}_{\pi}\left(M ; C_{0}, C_{1}\right)$.

Remark 5.6. This construction may be generalized to any Lie groupoid.

\section{Lagrangian Field Theories with Boundary}

As mentioned in Section 1.3, the constructions in this paper are related to the general ones for Lagrangian field theories on manifolds with boundary as in [4] and [5].

We start recalling a few facts. In general to a compact oriented manifold $N$ (possibly with boundary), of fixed dimension $d$, the theory associates a space of fields $\mathcal{F}_{N}$ and a function $S_{N}$ on $\mathcal{F}_{N}$ called the action functional. The case of this paper, fixing a manifold $M$ and a tensor $\pi$ on it, corresponds to $d=2, \mathcal{F}_{N}$ the space of bundle maps $T N \rightarrow T^{*} M$ and

$$
S_{N}(X, \eta)=\int_{N}\langle\eta, \mathrm{d} X\rangle+\frac{1}{2}\left\langle\eta, \pi^{\sharp} \eta\right\rangle
$$


where $X$ is a map $N \rightarrow M, \eta$ a section of $T^{*} N \otimes X^{*} T^{*} M$ and again $\langle, \quad\rangle$ is the canonical pairing between the cotangent and the tangent bundles to $M$. Notice that the action functional does not see the symmetric part of $\pi$, so it may be convenient to assume that $\pi$ is a bivector field. When $\pi$ is Poisson, this theory is called the Poisson sigma model [11,13]. We will call the general case the bivector sigma model (BSM).

Under certain assumptions (in particular locality), to a compact oriented ( $d-$ 1)-manifold $\Sigma$ the theory also associates an exact weak symplectic manifold $\left(\mathcal{F}_{\Sigma}^{\partial}, \Omega_{\Sigma}=\mathrm{d} \Theta_{\Sigma}\right)$ such that whenever $\Sigma=\partial M$ for a compact $d$-manifold $M$ there is a surjective submersion $\pi_{M}: \mathcal{F}_{M} \rightarrow \mathcal{F}_{\partial M}^{\partial}$. Denoting by $E L_{M}$ the zero set ${ }^{4}$ of the 1-form $\mathrm{EL}_{M}:=\mathrm{d} S_{M}-\pi_{M}^{*} \Theta_{\partial M}$, one obtains that the "evolution relation" $L_{M}:=$ $\pi_{M}\left(E L_{M}\right)$ is isotropic in $\mathcal{F}_{\partial M}^{\partial}$. In "good" theories the $L_{M} \mathrm{~s}$ should be Lagrangian. Observe that $L_{M}$ is here defined just as a subset; a good additional condition is that it should be a (possibly immersed) submanifold. One also has $\mathcal{F}_{\Sigma \sqcup \Sigma^{\prime}}^{\partial}=$ $\mathcal{F}_{\Sigma}^{\partial} \times \mathcal{F}_{\Sigma^{\prime}}^{\partial}$ as a product of exact weak symplectic manifolds and $\mathcal{F}_{\Sigma^{\mathrm{op}}}^{\partial}=\overline{\mathcal{F}_{\Sigma}^{\partial}}$, where $\Sigma^{\mathrm{op}}$ denotes $\Sigma$ with opposite orientation and bar denotes the same manifold with opposite one-form. Finally one defines the "space of Cauchy data" $\mathcal{C}_{\Sigma}$ as the space of points in $\mathcal{F}_{\Sigma}^{\partial}$ that can be completed to a pair of points in $L_{\Sigma \times[0, \epsilon]} \subset \mathcal{F}_{\Sigma}^{\partial} \times \overline{\mathcal{F}_{\Sigma}^{\partial}}$ for some $\epsilon>0$. Under some mild assumptions, one can show that, if $L_{\Sigma \times[0, \epsilon]}$ is Lagrangian for all $\epsilon$, then $\mathcal{C}_{\Sigma}$ is coisotropic. ${ }^{5}$ In the BSM, we get $\left(\mathcal{F}_{S^{1}}^{\partial}, \Theta_{S^{1}}\right)=$ $\left(T^{*} L M, \Theta\right)$ and $\mathcal{C}_{S^{1}}=\mathcal{C}_{\pi}(M)$ with the notations of Section 1.2. We have the following

THEOREM 6.1. $L_{S^{1} \times[0, \epsilon]}$ is an immersed Lagrangian submanifold $\forall \epsilon>0$ iff $\pi$ is Poisson.

Proof. If $L_{S^{1} \times[0, \epsilon]}$ is Lagrangian $\forall \epsilon>0$, then $\mathcal{C}_{S^{1}}=\mathcal{C}_{\pi}(M)$ is coisotropic by the general theory. By Theorem 1.5, $\pi$ is then Poisson.

\footnotetext{
${ }^{4}$ This is the set of solutions to Euler-Lagrange equations where one "ignores" the boundary.

${ }^{5}$ The first assumption is that the theory behaves well under diffeomorphisms: viz., a diffeomorphism of the bulk manifolds induces a diffeomorphism of the corresponding $E L$ spaces. This implies that each $L_{\Sigma \times[0, \epsilon]}$ is symmetric: $(x, y) \in L_{\Sigma \times[0, \epsilon]}$ iff $(y, x) \in L_{\Sigma \times[0, \epsilon]}$. The second assumption is locality which in particular implies that we can restrict solutions; so, if we know that $x$ lies in $\mathcal{C}_{\Sigma}$ because there is a $y$ with $(x, y) \in L_{\Sigma \times[0, \epsilon]}$, then we also know that for all $\epsilon^{\prime}<\epsilon$ there is a $y^{\prime}$ with $\left(x, y^{\prime}\right) \in L_{\Sigma \times\left[0, \epsilon^{\prime}\right]}$. (In the case of topological field theories, like the BSM, this part of the argument is much easier since $L_{\Sigma \times[0, \epsilon]}=L_{\Sigma \times\left[0, \epsilon^{\prime}\right]}$ for all $\epsilon, \epsilon^{\prime}$.)

Suppose now that $x$ lies in $\mathcal{C}_{\Sigma}$. Pick a compact neighborhood $U_{x}$ of $x$ in $\mathcal{C}_{\Sigma}$. For each $z$ in $U_{x}$ there is then an $\epsilon_{z}>0$ and a $y$ such that $(z, y) \in L_{\Sigma \times\left[0, \epsilon_{z}\right]}$. Let $\epsilon$ be the maximum $\epsilon_{z}$ for $z$ in $U_{x}$. Thanks to the locality assumption, we then have the simplified statement that for all $z \in U_{x}$ there is a $y_{z}$ such $\left(z, y_{z}\right) \in L_{\Sigma \times[0, \epsilon]}$. This in particular shows that $T_{x} \mathcal{C}_{\Sigma}$ consists of all $v \in T_{x} \mathcal{F}_{\Sigma}^{\partial}$ such that there is a $w \in T_{y} \mathcal{F}_{\Sigma}^{\partial}$, with $y:=y_{x}$, such that $(v, w) \in T_{(x, y)} L_{\Sigma \times[0, \epsilon]}$. Thanks to the symmetry property, we also see that $T_{(x, y)} L_{\Sigma \times[0, \epsilon]}$ is contained in $T_{x} \mathcal{C}_{\Sigma} \oplus T_{y} \mathcal{C}_{\Sigma}$. The orthogonal space of the latter, in $T_{x} \mathcal{F}_{\Sigma}^{\partial} \oplus \overline{T_{y} \mathcal{F}_{\Sigma}^{\partial}}$, is readily seen to be $\left(T_{x} \mathcal{C}_{\Sigma}\right)^{\perp} \oplus\left(T_{y} \mathcal{C}_{\Sigma}\right)^{\perp}$. Since $L_{\Sigma \times[0, \epsilon]}$ is Lagrangian by assumption, we conclude that $\left(T_{x} \mathcal{C}_{\Sigma}\right)^{\perp} \oplus\left(T_{y} \mathcal{C}_{\Sigma}\right)^{\perp}$ is contained in $T_{x} \mathcal{C}_{\Sigma} \oplus T_{y} \mathcal{C}_{\Sigma}$, so $T_{x} \mathcal{C}_{\Sigma}$ is coisotropic (and so is $T_{y} \mathcal{C}_{\Sigma}$ ). Since this can be shown for all $x \in \mathcal{C}_{\Sigma}$, we have that $\mathcal{C}_{\Sigma}$ is coisotropic.
} 
On the other hand, if $\pi$ is Poisson, then $\mathcal{C}_{S^{1}}=\mathcal{C}_{\pi}(M)$ is coisotropic by Theorem 1.5. In addition, $\forall \epsilon>0, L_{S^{1} \times[0, \epsilon]}$ consists of all pairs $\left(x, x^{\prime}\right) \in \mathcal{C}_{\pi}(M) \times \mathcal{C}_{\pi}(M)$ such that $x$ and $x^{\prime}$ are on the same characteristic leaf. ${ }^{6}$ This implies that $L_{S^{1} \times[0, \epsilon]}$ is an immersed Lagrangian submanifold. ${ }^{7}$

This story extends to the case when $\Sigma$ is only part of $\partial M$. If $\partial M=\Sigma \sqcup \Sigma^{\prime}$, then $\pi_{M}$ is the product of two surjective submersions $\pi_{M, \Sigma}$ and $\pi_{M, \Sigma^{\prime}}$ to $\mathcal{F}_{\Sigma}^{\partial}$ and $\mathcal{F}_{\Sigma^{\prime}}^{\partial}$, respectively. Upon picking a Lagrangian submanifold $L^{\prime}$ of $\mathcal{F}_{\Sigma^{\prime}}^{\partial}$ on which $\Theta_{\Sigma^{\prime}}$ vanishes, one sets $\mathcal{F}_{M}^{L^{\prime}}:=\pi_{M, \Sigma^{\prime}}^{-1}\left(L^{\prime}\right)$. Denoting by $S_{M}^{L^{\prime}}, \mathrm{EL}_{M}^{L^{\prime}}$ and $\pi_{M, \Sigma}^{L^{\prime}}$ the restrictions of $S_{M}, \mathrm{EL}_{M}$ and $\pi_{M, \Sigma}$ to $\mathcal{F}_{M}^{L^{\prime}}$, one then has $\mathrm{EL}_{M}^{L^{\prime}}=\mathrm{d} S_{M}^{L^{\prime}}-\left(\pi_{M, \Sigma}^{L^{\prime}, \Sigma}\right)^{*} \Theta_{\Sigma}$ and therefore $L_{M}^{L^{\prime}}:=\pi_{M, \Sigma}^{L^{\prime}}\left(E L_{M}^{L^{\prime}}\right)$ is isotropic, where $E L_{M}^{L^{\prime}}$ denotes the zero set of $E L_{M}^{L^{\prime}}$.

A further extension occurs when $M$ is a compact manifold with corners and the codimension-one boundary stratum of $M$, which we denote by $\partial M$, is the union of compact manifolds with boundary $\Sigma$ and $\Sigma^{\prime}$ joined along their common boundary, the codimension-two boundary stratum of $M$ (we assume that there are no further lower dimensional boundary strata). The story described in the previous paragraph extends verbatim to this case.

${ }^{6}$ The critical points of $S_{N}$ are solutions to

$$
\begin{aligned}
\mathrm{d} X & =-\pi^{\sharp}(X) \eta, \\
\mathrm{d} \eta_{i} & =\frac{1}{2} \partial_{i} \pi^{j k}(X) \eta_{j} \eta_{k} .
\end{aligned}
$$

For simplicity of notations we work in local coordinates (the rest of the computation may be done in covariant form since, using the first equation, the second can be written as $\partial \eta_{i}=$ $\left.\frac{1}{2}(\nabla \pi)_{i}^{j k}(X) \eta_{j} \eta_{k}\right)$. We write $\eta=\eta_{\|}-\beta \mathrm{d} t$, where $\eta_{\|}$is a 1 -form in the $S^{1}$ direction, $\beta$ a function and $t$ the coordinate on $[0, \epsilon]$. Writing $\mathrm{d}=\mathrm{d}_{\|}+\mathrm{d} t \delta$, with $d_{\|}$the differential in the $S^{1}$ direction and $\delta$ the partial derivative with respect to $t$, we may rewrite the equations as

$$
\begin{aligned}
\mathrm{d}_{\|} X & =-\pi^{\sharp}(X) \eta_{\|}, \\
\delta X & =\pi^{\sharp}(X) \beta, \\
\delta \eta_{\| i} & =-\mathrm{d}_{\|} \beta_{i}+\partial_{i} \pi^{j k}(X) \beta_{j} \eta_{\| k} .
\end{aligned}
$$

The first equation says that the restriction of $(X, \eta)$ to $S^{1} \times\{t\}$ for each $t$ yields an element of $\mathcal{C}_{\pi}(M)$. The two other equations say that $(X, \eta)$ evolves in the $t$ direction along the characteristic distribution of $\mathcal{C}_{\pi}(M)$, cf. (3.17).

${ }^{7}$ We follow the analogue proof in [9, Section 3.5.2]). Since $L_{S^{1} \times[0, \epsilon]}$ consists of pair of points on the same leaf of the characteristic distribution of $\mathcal{C}_{\pi}(M)$, it follows that it is an immersed submanifold. We now prove that it is Lagrangian. Let $(x, y)$ be in $L_{S^{1} \times[0, \epsilon]}$. Then, in addition to knowing that $T_{(x, y)} L_{S^{1} \times[0, \epsilon]}$ is a subspace of $T_{x} \mathcal{C}_{\pi}(M) \oplus T_{y} \mathcal{C}_{\pi}(M)$, which in turn is a coisotropic subspace of $T_{x}\left(T^{*} L M\right) \oplus \overline{T_{y}\left(T^{*} L M\right)}$, we now also know that it contains $\left(T_{x} \mathcal{C}_{\pi}(M)\right)^{\perp} \oplus$ $\left(T_{y} \mathcal{C}_{\pi}(M)\right)^{\perp}=\left(T_{x} \mathcal{C}_{\pi}(M) \oplus T_{y} \mathcal{C}_{\pi}(M)\right)^{\perp}$. Moreover, $T_{[x]} \mathcal{C}_{\pi}(M):=T_{x} \mathcal{C}_{\pi}(M) /\left(T_{x} \mathcal{C}_{\pi}(M)\right)^{\perp}$ gets canonically identified with $T_{y} \mathcal{C}_{\pi}(M) /\left(T_{y} \mathcal{C}_{\pi}(M)\right)^{\perp}$. Finally, $T_{(x, y)} L_{S^{1} \times[0, \epsilon]} /\left(T_{x} \mathcal{C}_{\pi}(M) \oplus T_{y} \mathcal{C}_{\pi}(M)\right)^{\perp}$ is the diagonal in $T_{[x]} \mathcal{C}_{\pi}(M) \oplus \overline{T_{[x]} \mathcal{C}_{\pi}(M)}$, which is Lagrangian. This proves that $T_{(x, y)} L_{S^{1} \times[0, \epsilon]}$ itself is Lagrangian, see [4, Proposition A.1(3)]. 
Finally, one can define the "space of Cauchy data" $\mathcal{C}_{\Sigma}^{L^{\prime}}$ as the space of points in $\mathcal{F}_{\Sigma}^{\partial}$ that can be completed to a pair of points in $L_{\Sigma \times[0, \epsilon]}^{L^{\prime}} \subset \mathcal{F}_{\Sigma}^{\partial} \times \overline{\mathcal{F}_{\Sigma}^{\partial}}$ for some $\epsilon>0$, where $L^{\prime}$ is a fixed Lagrangian submanifold of $\mathcal{F}_{\partial \Sigma \times[0, \epsilon]}{ }^{8}$ Again, one shows that, if $L_{\Sigma \times[0, \epsilon]}^{L^{\prime}}$ is Lagrangian for all $\epsilon$, then $\mathcal{C}_{\Sigma}^{L^{\prime}}$ is coisotropic.

In the BSM, we get $\left(\mathcal{F}_{I}^{\partial}, \Theta_{I}\right)=\left(T^{*} P M, \Theta\right)$ with the notations of the Introduction. Moreover, for a submanifold $C$ of $M$, the space $L_{C}$ of bundle maps $T[0, \epsilon] \rightarrow N^{*} C$ is a Lagrangian submanifold of $\mathcal{F}_{[0, \epsilon]}^{\partial}$. Finally, one has $\mathcal{C}_{I}^{L_{C_{0}} \times L_{C_{1}}}=$ $\mathcal{C}_{\pi}\left(M ; C_{0}, C_{1}\right)$ and

THEOREM 6.2. $L_{I \times[0, \epsilon]}^{L_{M} \times L_{M}}$ is an immersed Lagrangian submanifold $\forall \epsilon>0$ iff $\pi$ is Poisson. If $\pi$ is Poisson and $C_{0}$ and $C_{1}$ are coisotropic, then $L_{I \times[0, \epsilon]}^{L_{C_{0}} \times L_{C_{1}}}$ is Lagrangian $\forall \epsilon>0$.

The proof is similar to the case of $S^{1}$ but now uses Theorem 1.1, Corollary 1.3 and the results of Section 3.2.1.

\section{Acknowledgements}

I thank I. Contreras for useful discussions and comments.

\section{References}

1. Calvo, I., Falceto, F.: Poisson reduction and branes in Poisson sigma models. Lett. Math. Phys. 70, 231-247 (2004)

2. Cattaneo, A.S.: On the integration of Poisson manifolds, Lie algebroids, and coisotropic submanifolds. Lett. Math. Phys 67, 33-48 (2004)

3. Cattaneo, A.S., Contreras, I.: Groupoids and Poisson sigma models with boundary. http://arxiv.org/abs/1206.4330 (arXiv:1206.4330). In: Geometric, Algebraic and Topological Methods for Quantum Field Theory, Proceedings of the 7th Villa de Leyva Summer School, World Scientific (2013, to appear)

4. Cattaneo, A.S., Mnëv P., Reshetikhin, N.: Classical BV theories on manifolds with boundaries. http://arxiv.org/abs/1201.0290 (math-ph/1201.0290)

5. Cattaneo, A.S., Mnëv, P., Reshetikhin, N.: Classical and quantum Lagrangian field theories with boundary. http://pos.sissa.it/archive/conferences/155/044/CORFU2011_04 4.pdf (PoS(CORFU2011)044), http://arxiv.org/abs/1207.0239 (arXiv:1207.0239)

6. Cattaneo, A.S., Felder, G.: Poisson sigma models and symplectic groupoids. In: Landsman, N.P., Pflaum, M., Schlichenmeier, M. (eds.) Quantization of Singular Symplectic Quotients, Progress in Mathematics, vol. 198. Birkhauser, Basel, pp. 61-93 (2001)

7. Cattaneo, A.S., Felder, G.: Coisotropic submanifolds in Poisson geometry and branes in the Poisson sigma model. Lett. Math. Phys. 69, 157-175 (2004)

8. Cattaneo, A.S., Zambon, M.: Pre-Poisson submanifolds. Travaux mathématiques 17, 61-74 (2007)

\footnotetext{
${ }^{8}$ Since a diffeomorphism $[0, \epsilon] \rightarrow\left[0, \epsilon^{\prime}\right]$ induces a symplectomorphism $\mathcal{F}_{\gamma \times[0, \epsilon]}^{\partial} \rightarrow \mathcal{F}_{\gamma \times\left[0, \epsilon^{\prime}\right]}^{\partial}$, it is enough to select $L^{\prime}$ in $\mathcal{F}_{\gamma \times[0, \epsilon]}$ for a fixed $\epsilon$.
} 
9. Contreras, I.: Relational Symplectic Groupoids and Poisson Sigma Models with Boundary, Ph. D. thesis (Zurich, 2013), http://arxiv.org/abs/1306.4119

10. Crainic, M., Fernandes, R.L.: Integrability of Lie brackets. Ann. Math. 157, 575620 (2003)

11. Ikeda, N.: Two-dimensional gravity and nonlinear gauge theory. Ann. Phys. 235, 435464 (1994)

12. Landsman, N.P.: Quantized reduction as a tensor product. In: Landsman, N.P., Pflaum, M., Schlichenmeier, M. (eds.) Quantization of Singular Symplectic Quotients, Progress in Mathematics, vol. 198. Birkhäuser, Basel, pp. 137-180 (2001)

13. Schaller, P., Strobl, T.: Poisson structure induced (topological) field theories. Modern Phys. Lett. A 9, 3129-3136 (1994)

14. Weinstein, A.: The local structure of Poisson manifolds. J. Differ. Geom 18, 523557 (1983) 